\title{
Stochastic differential games with asymmetric information.
}

\author{
Pierre Cardaliaguet and Catherine Rainer \\ Département de Mathématiques, Université de Bretagne Occidentale, \\ 6, avenue Victor-le-Gorgeu, B.P. 809, 29285 Brest cedex, France \\ e-mail : Pierre.Cardaliaguet@univ-brest.fr, Catherine.Rainer@univ-brest.fr
}

August 20, 2021

\begin{abstract}
We investigate a two-player zero-sum stochastic differential game in which the players have an asymmetric information on the random payoff. We prove that the game has a value and characterize this value in terms of dual solutions of some second order Hamilton-Jacobi equation.
\end{abstract}

Key-words : stochastic differential game, asymmetric information, viscosity solution.

A.M.S. classification : 49N70, 49L25, 91A23.

\section{Introduction}

This paper is devoted to a class of two-player zero-sum stochastic differential game in which the players have different information on the payoff. In this basic model, the terminal cost is chosen (at the initial time) randomly among a finite set of costs $\left\{g_{i j}, i \in\{1, \ldots, I\}, j \in\right.$ $\{1, \ldots, J\}\}$. More precisely, the indexes $i$ and $j$ are chosen independently according to a probability $p \otimes q$ on $\{1, \ldots, I\} \times\{1, \ldots, J\}$. Then the index $i$ is announced to the first player and the index $j$ to the second player. The players control the stochastic differential equation

$$
\begin{aligned}
& d X_{s}=b\left(s, X_{s}, u_{s}, v_{s}\right) d s+\sigma\left(s, X_{s}, u_{s}, v_{s}\right) d B_{s}, s \in[t, T], \\
& X_{t}=x,
\end{aligned}
$$

through their respective controls $\left(u_{s}\right)$ and $\left(v_{s}\right)$ in order, for the first player, to minimize $E\left[g_{i j}\left(X_{T}\right)\right]$ and, for the second player, to maximize this quantity. Note that the players do not really know which payoff they are actually optimizing because the first player, for 
instance, ignores which index $j$ has been chosen. The key assumption in our model is that the players observe the evolving state $\left(X_{s}\right)$. So they can deduce from this observation the behavior of their opponent and try to derive from it some knowledge on their missing data.

The formalization of such a game is quite involved: we refer to the second section of the paper where the notations are properly defined. In order to describe our results, let us introduce the upper and lower value functions $V^{+}$and $V^{-}$of the game:

$$
\begin{aligned}
V^{+}(t, x, p, q) & =\inf _{\hat{\alpha} \in\left(\mathcal{A}_{r}(t)\right)^{I}} \sup _{\left.\hat{\beta} \in \mathcal{B}_{r}(t)\right)^{J}} J^{p, q}(t, x, \hat{\alpha}, \hat{\beta}), \\
V^{-}(t, x, p, q) & =\sup _{\hat{\beta} \in\left(\mathcal{B}_{r}(t)\right)^{J}} \inf _{\hat{\alpha} \in\left(\mathcal{A}_{r}(t)\right)^{I}} J^{p, q}(t, x, \hat{\alpha}, \hat{\beta}) .
\end{aligned}
$$

where $J^{p, q}(t, x, \hat{\alpha}, \hat{\beta})$ is the expectation under the probability $p \otimes q$ of the payoff associated with the strategies $\hat{\alpha}=\left(\alpha_{i}\right)_{i \in\{1, \ldots, I\}}$ and $\hat{\beta}=\left(\beta_{j}\right)_{j \in\{1, \ldots, J\}}$ of the players. The strategy $\hat{\alpha}$ takes into account the knowledge by the first player of the index $i$ while $\hat{\beta}$ takes into account the knowledge of $j$ by the second player. Our main result is that, under Isaacs'condition, the two value functions coincide: $V^{+}=V^{-}$. Moreover, $\mathbf{V}:=V^{+}=V^{-}$is the unique viscosity solution in the dual sense of some second order Hamilton-Jacobi equation. This means that

(i) $\mathbf{V}$ is convex with respect to $p$ and concave with respect to $q$,

(ii) the convex conjugate of $\mathbf{V}$ with respect to $p$ is a subsolution of some Hamilton-JacobiIsaacs (HJI) equation in the viscosity sense,

(iii) the concave conjugate of $\mathbf{V}$ with respect to $q$ is a supersolution of a symmetric HJI equation,

(iv) $\mathbf{V}(T, x, p, q)=\sum_{i, j} p_{i} q_{j} g_{i j}(x)$ where $p=\left(p_{i}\right)_{i \in\{1, \ldots, I\}}$ and $q=\left(q_{j}\right)_{j \in\{1, \ldots, J\}}$.

We strongly underline that in general the value functions are not solution of the standard HJI equation: indeed $\mathbf{V}$ does not satisfy a dynamic programming principle in a classical sense.

An important current in Mathematical Finance is the modeling of insider trading (see for example Amendinger, Becherer, Schweizer [2] or Corcuera, Imkeller, Kohatsu-Higa, Nualart [7] and references therein). The basic question studied in these works is to evaluate how the addition of knowledge for a trader - i.e., mathematically, the addition to the original filtration of a variable depending on the future - shows up in his investing strategies, and an important tool is the theory of enlargement of filtrations. Our approach is completely 
different. Indeed, what is important in our game is not that the players have "more" information than what is contained in the filtration of the Brownian motion, but that their information differs from that of their opponent. In some sense we try to understand the strategic role of information in the game.

The model described above is strongly inspired by a similar one studied by Aumann and Maschler in the framework of repeated games. Since their seminal papers (reproduced in [3]), this model has attracted a lot of attention in game theory (see [11, [13, [15], [16]). However it is only recently that the first author has adapted the model to deterministic differential games (see [5], [6]).

The aim of this paper is to generalize the results of [5] to stochastic differential games and to game with integral payoffs. There are several difficulties towards this aim. First the notion of strategies for stochastic differential games is quite intricated (see [12, [14]). For our game it is all the more difficult that the players have to introduce additional noise in their strategies in order to confuse their oponent. One of the achievements of this paper is an important simplification of the notion of strategy which allows the introduction of the notion of random strategies. This also simplifies several proofs of [5]. Second the existence of a value for "classical" stochastic differential games relies on a comparison principle for some second order Hamilton-Jacobi equations. Here we have to be able to compare functions satisfying the condition (i,ii,iv) defined above with functions satisfying (i,iii,iv). While for deterministic differential games (i.e., first order HJI equations) we could do this without too much trouble (see [5]), for stochastic differential games (i.e., second order HJI equations) the proof is much more involved. In particular it requires a new maximum principle for lower semicontinuous functions (see the appendix) which is the most technical part of the paper.

The paper is organized in the following way: in section 2 , we introduce the main notations and the notion of random strategies and we define the value functions of our game. In section 3 we prove that the value functions (and more precisely the convex and concave conjugates) are sub- and supersolutions of some HJ equation. Section 4 is devoted to the comparison principle and to the existence of the value. In Section 5 we investigate stochastic differential games with a running cost. The appendix is devoted to a new maximum principle. 


\section{Definitions.}

\subsection{The dynamics.}

Let $T>0$ be a fixed finite time horizon. For $(t, x) \in[0, T] \times \mathbb{R}^{n}$, we consider the following doubly controlled stochastic system :

$$
\begin{aligned}
& d X_{s}=b\left(s, X_{s}, u_{s}, v_{s}\right) d s+\sigma\left(s, X_{s}, u_{s}, v_{s}\right) d B_{s}, s \in[t, T] \\
& X_{t}=x
\end{aligned}
$$

where $B$ is a $d$-dimensional standard Brownian motion on a given probability space $(\Omega, \mathcal{F}, P)$. For $s \in[t, T]$, we set

$$
\mathcal{F}_{t, s}=\sigma\left\{B_{r}-B_{t}, r \in[t, s]\right\} \vee \mathcal{P},
$$

where $\mathcal{P}$ is the set of all null-sets of $P$.

The processes $u$ and $v$ are assumed to take their values in some compact metric spaces $U$ and $V$ respectively. We suppose that the functions $b:[0, T] \times \mathbb{R}^{n} \times U \times V \rightarrow \mathbb{R}^{n}$ and $\sigma:[0, T] \times \mathbb{R}^{n} \times U \times V \rightarrow \mathbb{R}^{n \times d}$ are continuous and satisfy the assumption $(\mathrm{H})$ :

(H) $b$ and $\sigma$ are bounded and Lipschitz continuous with respect to $(t, x)$, uniformly in $(u, v) \in U \times V$.

We also assume Isaacs' condition : for all $(t, x) \in[0, T] \times \mathbb{R}^{n}, p \in \mathbb{R}^{n}$, and all $A \in \mathcal{S}_{n}$ (where $\mathcal{S}_{n}$ is the set of symmetric $n \times n$ matrices) holds:

$$
\begin{aligned}
\inf _{u} \sup _{v}\left\{<b(t, x, u, v), p>+\frac{1}{2} \operatorname{Tr}\left(A \sigma(t, x, u, v) \sigma^{*}(t, x, u, v)\right)\right\}= \\
\sup _{v} \inf _{u}\left\{<b(t, x, u, v), p>+\frac{1}{2} \operatorname{Tr}\left(A \sigma(t, x, u, v) \sigma^{*}(t, x, u, v)\right)\right\}
\end{aligned}
$$

We set $H(t, x, p, A)=\inf _{u} \sup _{v}\left\{\langle b(t, x, u, v), p\rangle+\frac{1}{2} \operatorname{Tr}\left(A \sigma(t, x, u, v) \sigma^{*}(t, x, u, v)\right)\right\}$.

For $t \in[0, T)$, we denote by $\mathcal{C}\left([t, T], \mathbb{R}^{n}\right)$ the set of continuous maps from $[t, T]$ to $\mathbb{R}^{n}$.

\subsection{Admissible controls.}

Definition 2.1 An admissible control u for player I (resp. II) on $[t, T]$ is a process taking values in $U$ (resp. $V$ ), progressively measurable with respect to the filtration $\left(\mathcal{F}_{t, s}, s \geq t\right)$. The set of admissible controls for player I (resp. II) on $[t, T]$ is denoted by $\mathcal{U}(t)($ resp. $\mathcal{V}(t))$.

We identify two processes $u$ and $\bar{u}$ in $\mathcal{U}(t)$ if $P\{u=\bar{u}$ a.e. in $[t, T]\}=1$.

Under assumption $(\mathrm{H})$, for all $(t, x) \in[0, T] \times \mathbb{R}^{n}$ and $(u, v) \in \mathcal{U}(t) \times \mathcal{V}(t)$, there exists a unique solution to (2.1) that we denote by $X^{t, x, u, v}$. 


\subsection{Strategies.}

Definition 2.2 A strategy for player I starting at time $t$ is a Borel-measurable map $\alpha$ : $[t, T] \times \mathcal{C}\left([t, T], \mathbb{R}^{n}\right) \rightarrow U$ for which there exists $\delta>0$ such that, $\forall s \in[t, T], f, f^{\prime} \in$ $\mathcal{C}\left([t, T], \mathbb{R}^{n}\right)$, if $f=f^{\prime}$ on $[t, s]$, then $\alpha(\cdot, f)=\alpha\left(\cdot, f^{\prime}\right)$ on $[t, s+\delta]$.

We define strategies for player II in a symmetric way and denote by $\mathcal{A}(t)($ resp. $\mathcal{B}(t))$ the set of strategies for player I (resp. player II).

We have the following existence result :

Lemma 2.1 For all $(t, x)$ in $[0, T] \times \mathbb{R}^{n}$, for all $(\alpha, \beta) \in \mathcal{A}(t) \times \mathcal{B}(t)$, there exists a unique couple of controls $(u, v) \in \mathcal{U}(t) \times \mathcal{V}(t)$ that satisfies $P$-a.s.

$$
(u, v)=\left(\alpha\left(\cdot, X^{t, x, u, v}\right), \beta\left(\cdot, X^{t, x, u, v}\right)\right) \text { on }[t, T] .
$$

Proof: The controls $u$ and $v$ will be built step by step. Let $\delta>0$ be a common delay for $\alpha$ and $\beta$. We can choose $\delta$ such that $T=t+N \delta$ for some $N \in \mathbb{N}^{*}$.

By definition, on $[t, t+\delta)$, for all $f \in \mathcal{C}\left([t, T], \mathbb{R}^{n}\right), \alpha(s, f)=\alpha(s, f(t))$. Since, for all $(u, v) \in \mathcal{U}(t) \times \mathcal{V}(t), X_{t}^{t, x, u, v}=x$, the control $u$ is uniquely defined on $[t, t+\delta)$ by

$$
\forall s \in[t, t+\delta), u(s)=\alpha(s, x)
$$

The same holds for $v$, what permits us to define the process $X^{t, x, u, v}$ on $[t, t+\delta)$ as a solution of the system (2.1) restricted on the interval $[t, t+\delta)$.

Now suppose that $u, v$ and $X^{t, x, u, v}$ are $P$-a.s. defined uniquely on some interval $[t, t+k \delta)$, $k \in\{1, \ldots, N-1\}$. This allows us to set,

$$
\forall s \in[t+k \delta, t+(k+1) \delta), u_{s}=\alpha\left(s, X^{t, x, u^{k}, v^{k}}\right), v_{s}=\beta\left(s, X^{t, x, u^{k}, v^{k}}\right),
$$

where

$$
\left(u^{k}, v^{k}\right)=\left\{\begin{array}{l}
(u, v) \text { on }[t, t+k \delta) \\
\left(u_{0}, v_{0}\right) \text { else },
\end{array}\right.
$$

for some arbitrary $\left(u_{0}, v_{0}\right) \in \mathcal{U}(t) \times \mathcal{V}(t)$.

Considering $X^{t, x, u^{k}, v^{k}}$ as a random variable with values in the set of paths $\mathcal{C}\left([t, T), \mathbb{R}^{n}\right)$, it is clear that the map $(s, \omega) \rightarrow u_{s}(\omega)$ (defined on $[t+k \delta, t+(k+1) \delta) \times \Omega$ ) as the composition of the Borel measurable application $\alpha$ with the map $(s, \omega) \rightarrow\left(s, X^{t, x, u^{k}, v^{k}}(\omega)\right)$, is a process on $[t+k \delta, t+(k+1) \delta)$ with measurable paths. Further, the non anticipativity of $\alpha$ guaranties that, for all $s \in[t+k \delta, t+(k+1) \delta), u_{s}$ is $\mathcal{F}_{t, t+k \delta}$-measurable and the process $\left.u\right|_{[t, t+(k+1) \delta)}$ is $\left(\mathcal{F}_{t, s}\right)$-progressively measurable. The same holds of course for $\left.v\right|_{[t, t+(k+1) \delta)}$. 
With $(u, v)$ defined on $[t, t+(k+1) \delta)$, we can now define the process $X^{t, x, u, v}$ up to time $t+(k+1) \delta$. This completes the proof by induction.

We denote by $X^{t, x, \alpha, \beta}$ the process $X^{t, x, u, v}$, with $(u, v)$ associated to $(\alpha, \beta)$ by relation (2.3).

In the frame of incomplete information it is necessary to introduce random strategies. In contrast with [5] and [6], where the random probabilities are supposed to be absolutely continuous with respect to the Lebesgue measure, play a random strategy will consist here to choose some strategy in a finite set of possibilities, i.e. the involved probabilities are finite. It is not clear if this assumption is more realistic nor if the notation will be lighter, nevertheless this alternative allows us to avoid some technical steps of measure theory, in a paper that is already technical enough.

Notation: For $R \in \mathbb{N}^{*}$, let $\Delta(R)$ be the set of all $\left(r_{1}, \ldots, r_{R}\right) \in[0,1]^{R}$ that satisfy $\sum_{n=1}^{R} r_{n}=1$.

We define a random strategy $\bar{\alpha}$ for player I by $\bar{\alpha}=\left(\alpha^{1}, \ldots \alpha^{R} ; r^{1}, \ldots, r^{R}\right)$, with $R \in \mathbb{N}^{*}$, $\left(\alpha^{1}, \ldots \alpha^{R}\right) \in(\mathcal{A}(t))^{R},\left(r^{1}, \ldots, r^{R}\right) \in \Delta(R)$.

The heuristic interpretation of $\bar{\alpha}$ is that player I's strategy amounts to choose the pure strategy $\alpha^{k}$ with probability $r^{k}$.

We define in a similar way the random strategies for player II, and denote by $\mathcal{A}_{r}(t)$ (resp. $\left.\mathcal{B}_{r}(t)\right)$ the set of all random strategies for player I (resp. player II).

Finally, identifying $\alpha \in \mathcal{A}(t)$ with $(\alpha ; 1) \in \mathcal{A}_{r}(t)$, we can write $\mathcal{A}(t) \subset \mathcal{A}_{r}(t)$, and the same holds for $\mathcal{B}(t)$ and $\mathcal{B}_{r}(t)$.

\subsection{The payoff.}

Fix $I, J \in \mathbb{N}^{*}$.

For $1 \leq i \leq I, 1 \leq j \leq J$, let $g_{i j}: \mathbb{R}^{n} \rightarrow \mathbb{R}$ be the terminal payoffs. We assume that

For $1 \leq i \leq I, 1 \leq j \leq J, g_{i j}$ are Lipschitz continuous and bounded.

For $(p, q) \in \Delta(I) \times \Delta(J)$, with $p=\left(p_{1}, \ldots, p_{I}\right), q=\left(q_{1}, \ldots q_{J}\right)$, we denote with a hat the elements of $\left(\mathcal{A}_{r}(t)\right)^{I}\left(\operatorname{resp} .\left(\mathcal{B}_{r}(t)\right)^{J}\right): \hat{\alpha}=\left(\bar{\alpha}_{1}, \ldots, \bar{\alpha}_{I}\right), \hat{\beta}=\left(\bar{\beta}_{1}, \ldots, \bar{\beta}_{J}\right)$.

We adopt following notations : 
For fixed $(i, j) \in\{1, \ldots, I\} \times\{1, \ldots, J\}$ and strategies $(\alpha, \beta) \in \mathcal{A}(t) \times \mathcal{B}(t)$, the payoff of the game with only one possible terminal payoff function $g_{i j}$ will be denoted by

$$
J_{i j}(t, x, \alpha, \beta)=E\left[g_{i j}\left(X_{T}^{t, x, \alpha, \beta}\right)\right] .
$$

Now let $(\bar{\alpha}, \bar{\beta}) \in \mathcal{A}_{r}(t) \times \mathcal{B}_{r}(t)$ be two random strategies, with $\bar{\alpha}=\left(\alpha^{1}, \ldots, \alpha^{R} ; r^{1}, \ldots, r^{R}\right)$ and $\bar{\beta}=\left(\beta^{1}, \ldots, \beta^{S} ; s^{1}, \ldots, s^{S}\right)$. The payoff associated with the pair $\left.(\bar{\alpha}, \bar{\beta}) \in \mathcal{A}_{r}(t) \times \mathcal{B}_{r}(t)\right)$, is the average of the payoffs with respect to the probability distributions associated to the strategies:

$$
J_{i j}(t, x, \bar{\alpha}, \bar{\beta})=\sum_{k=1}^{R} \sum_{l=1}^{S} r^{k} s^{l} E\left[g_{i j}\left(X_{T}^{t, x, \alpha^{k}, \beta^{l}}\right)\right] .
$$

Further, for $p \in \Delta(I), j \in\{1, \ldots, J\}, \hat{\alpha} \in\left(\mathcal{A}_{r}(t)\right)^{I}$ and $\bar{\beta} \in \mathcal{B}_{r}(t)$ we will use the notation

$$
J_{j}^{p}(t, x, \hat{\alpha}, \bar{\beta})=\sum_{i=1}^{I} p_{i} J_{i j}\left(t, x, \bar{\alpha}_{i}, \bar{\beta}\right)=\sum_{i=1}^{I} p_{i} \sum_{k, l} r^{k} s^{l} E\left[g_{i j}\left(X_{T}^{t, x, \alpha_{i}^{k}, \beta^{l}}\right)\right] .
$$

A symmetric notation holds for $\bar{\alpha} \in \mathcal{A}_{r}(t)$ and $\hat{\beta} \in\left(\mathcal{B}_{r}(t)\right)^{J}$. Finally, the payoff of the game is, for $(\hat{\alpha}, \hat{\beta}) \in\left(\mathcal{A}_{r}(t)\right)^{I} \times\left(\mathcal{B}_{r}(t)\right)^{J}, p \in \Delta(I), q \in \Delta(J)$,

$$
J^{p, q}(t, x, \hat{\alpha}, \hat{\beta})=\sum_{i=1}^{I} \sum_{j=1}^{J} p_{i} q_{j} J_{i j}\left(t, x, \bar{\alpha}_{i}, \bar{\beta}_{j}\right) .
$$

The reference to $(t, x)$ in the notations is dropped when there is no possible confusion : we will write $J_{i j}(\alpha, \beta), J_{i j}(\bar{\alpha}, \beta), \ldots$

We define the value functions for the game by

$$
\begin{aligned}
V^{+}(t, x, p, q) & =\inf _{\hat{\alpha} \in\left(\mathcal{A}_{r}(t)\right)^{I} \sup _{\left.\hat{\beta} \in \mathcal{B}_{r}(t)\right)^{J}} J^{p, q}(t, x, \hat{\alpha}, \hat{\beta}),} \\
V^{-}(t, x, p, q) & =\sup _{\hat{\beta} \in\left(\mathcal{B}_{r}(t)\right)^{J}} \inf _{\hat{\alpha} \in\left(\mathcal{A}_{r}(t)\right)^{I}} J^{p, q}(t, x, \hat{\alpha}, \hat{\beta}) .
\end{aligned}
$$

Again we will write $V^{+}(p, q)$ and $V^{-}(p, q)$ if there is no possible confusion on $(t, x)$.

The following lemma follows easily from classical estimations for stochastic differential equations :

Lemma 2.2 $V^{+}$and $V^{-}$are bounded, Lipschitz continuous with respect to $x, p, q$ and Hölder continuous with respect to $t$.

Following [3] we now state one of the basic properties of the value functions. The technique of proof of this statement is known as the splitting method in repeated game theory (see [3], [16]). 
Proposition 2.1 For all $(t, x) \in[0, T] \times \mathbb{R}^{n}$, the maps $(p, q) \rightarrow V^{+}(t, x, p, q)$ and $(p, q) \rightarrow$ $V^{-}(t, x, p, q)$ are convex in $p$ and concave in $q$.

Proof: We only prove the result for $V^{+}$, the proof for $V^{-}$is the same. First $V^{+}$can be rewritten as

$$
V^{+}(p, q)=\inf _{\hat{\alpha} \in\left(\mathcal{A}_{r}(t)\right)^{I}} \sum_{j=1}^{J} q_{j} \sup _{\bar{\beta} \in \mathcal{B}_{r}(t)} J_{j}^{p}(\hat{\alpha}, \bar{\beta}) .
$$

It follows that $V^{+}$is concave in $q$.

Now fix $q \in \Delta(J)$ and let $p, p^{\prime} \in \Delta(I)$ and $a \in(0,1)$. Without loss of generality we can assume that, for all $i \in\{1, \ldots, I\}, p_{i}$ and $p_{i}^{\prime}$ are not simultaneously equal to zero.

We get a new element of $\Delta(I)$ if we set $p^{a}=a p+(1-a) p^{\prime}$. For $\epsilon>0$, let $\hat{\alpha} \in\left(\mathcal{A}_{r}(t)\right)^{I}$ be $\epsilon$-optimal for $V^{+}(p, q)$ (resp. $\hat{\alpha}^{\prime} \in\left(\mathcal{A}_{r}(t)\right)^{I} \epsilon$-optimal for $\left.V^{+}\left(p^{\prime}, q\right)\right)$.

We define a new strategy $\hat{\alpha}^{a}=\left(\bar{\alpha}_{1}^{a}, \ldots, \bar{\alpha}_{I}^{a}\right)$ by

$$
\bar{\alpha}_{i}^{a}=\left(\alpha_{i}^{1}, \ldots, \alpha_{i}^{R}, \alpha_{i}^{\prime 1}, \ldots, \alpha_{i}^{\prime R^{\prime}} ;\left(r_{i}^{a}\right)^{1}, \ldots,\left(r_{i}^{a}\right)^{\left(R+R^{\prime}\right)}\right), i \in\{1, \ldots, I\}
$$

with

$$
\left(r_{i}^{a}\right)^{k}= \begin{cases}\frac{a p_{i}}{p_{i}^{a}} r_{i}^{k} & \text { for } k \in\{1, \ldots, R\} \\ \frac{(1-a) p_{i}^{\prime}}{p_{i}^{a}} r_{i}^{\prime k-R} & \text { for } k \in\left\{R+1, \ldots, R+R^{\prime}\right\}\end{cases}
$$

(it is easy to check that $\left.\hat{\alpha}^{a} \in\left(\mathcal{A}_{r}(t)\right)^{I}\right)$.

This means that, for all $\hat{\beta} \in\left(\mathcal{B}_{r}(t)\right)^{J}$,

$$
J^{p^{a}, q}\left(\hat{\alpha}^{a}, \hat{\beta}\right)=\sum_{i=1}^{I}\left\{a p_{i} \sum_{k=1}^{R} r_{i}^{k} J_{i}^{q}\left(\alpha_{i}^{k}, \hat{\beta}\right)+(1-a) p_{i}^{\prime} \sum_{k=1}^{R^{\prime}} r_{i}^{\prime k} J_{i}^{q}\left(\alpha_{i}^{\prime k}, \hat{\beta}\right)\right\}
$$

Thus

$$
\sup _{\hat{\beta} \in\left(\mathcal{B}_{r}(t)\right)^{J}} J^{p^{a}, q}\left(\hat{\alpha}^{a}, \hat{\beta}\right) \leq a \sup _{\hat{\beta} \in\left(\mathcal{B}_{r}(t)\right)^{J}} J^{p, q}(\hat{\alpha}, \hat{\beta})+(1-a) \sup _{\hat{\beta} \in\left(\mathcal{B}_{r}(t)\right)^{J}} J^{p^{\prime}, q}\left(\hat{\alpha}^{\prime}, \hat{\beta}\right) .
$$

It follows by the choice of $\hat{\alpha}$ and $\hat{\alpha}^{\prime}$ that

$$
V^{+}\left(p^{a}, q\right) \leq a V^{+}(p, q)+(1-a) V^{+}\left(p^{\prime}, q\right)
$$




\section{Subdynamic programming and Hamilton-Jacobi-Bellman equations for the Fenchel conjugates.}

Since $V^{+}$and $V^{-}$are convex with respect to $p$ and concave with respect to $q$, it is natural to introduce the Fenchel conjugates of these functions. For this we use the following notations. For any $w:[0, T] \times \mathbb{R}^{n} \times \Delta(I) \times \Delta(J) \rightarrow \mathbb{R}$, we define the Fenchel conjugate $w^{*}$ of $w$ with respect to $p$ by

$$
w^{*}(t, x, \hat{p}, q)=\sup _{p \in \Delta(I)}\{\langle\hat{p}, p\rangle-w(t, x, p, q)\},(t, x, \hat{p}, q) \in[0, T] \times \mathbb{R}^{n} \times \mathbb{R}^{I} \times \Delta(J) .
$$

For $w$ defined on the dual space $[0, T] \times \mathbb{R}^{n} \times \mathbb{R}^{I} \times \Delta(J)$, we also set

$$
w^{*}(t, x, p, q)=\sup _{\hat{p} \in \mathbb{R}^{I}}\{\langle\hat{p}, p\rangle-w(t, x, \hat{p}, q)\},(t, x, p, q) \in[0, T] \times \mathbb{R}^{n} \times \Delta(I) \times \Delta(J) .
$$

It is well known that, if $w$ is convex, we have $\left(w^{*}\right)^{*}=w$.

We also have to introduce the concave conjugate with respect to $q$ of a map $w:[0, T] \times$ $\mathbb{R}^{n} \times \Delta(I) \times \Delta(J) \rightarrow \mathbb{R}:$

$$
w^{\sharp}(t, x, p, \hat{q})=\inf _{q \in \Delta(J)}\{\langle\hat{q}, q\rangle-w(t, x, p, q)\},(t, x, p, \hat{q}) \in[0, T] \times \mathbb{R}^{n} \times \Delta(I) \times \mathbb{R}^{J} .
$$

We use the following notations for the sub- and superdifferentials with respect to $\hat{p}$ and $\hat{q}$ respectively: if $w:[0, T] \times \mathbb{R}^{n} \times \mathbb{R}^{I} \times \Delta(J) \rightarrow \mathbb{R}$, we set

$$
\partial_{\hat{p}}^{-} w(t, x, \hat{p}, q)=\left\{p \in \mathbb{R}^{I}, w(t, x, \hat{p}, q)+\left\langle p, \hat{p}^{\prime}-\hat{p}\right\rangle \leq w\left(t, x, \hat{p}^{\prime}, q\right) \forall \hat{p}^{\prime} \in \mathbb{R}^{I}\right\}
$$

and if $w:[0, T] \times \mathbb{R}^{n} \times \Delta(I) \times \mathbb{R}^{J} \rightarrow \mathbb{R}$

$$
\partial_{\hat{q}}^{+} w(t, x, p, \hat{q})=\left\{q \in \mathbb{R}^{J}, w(t, x, p, \hat{q})+\left\langle q, \hat{q}^{\prime}-\hat{q}\right\rangle \geq w\left(t, x, p, \hat{q}^{\prime}\right) \forall \hat{q}^{\prime} \in \mathbb{R}^{J}\right\} .
$$

In this chapter, we will show that $V^{+\sharp}$ and $V^{-*}$ satisfy a subdynamic programming property. This part follows several ideas of [10], [11].

Lemma 3.1 (Reformulation of $V^{-*}$ )

For all $(t, x, \hat{p}, q) \in[0, T] \times \mathbb{R}^{n} \times \mathbb{R}^{I} \times \Delta(J)$, we have

$$
V^{-*}(t, x, \hat{p}, q)=\inf _{\hat{\beta} \in\left(\mathcal{B}_{r}(t)\right)^{J}} \sup _{\alpha \in \mathcal{A}(t)} \max _{i \in\{1, \ldots, I\}}\left\{\hat{p}_{i}-J_{i}^{q}(t, x, \alpha, \hat{\beta})\right\} .
$$

Proof. We begin to establish a first expression for $V^{-*}$ :

$$
V^{-*}(\hat{p}, q)=\inf _{\hat{\beta} \in\left(\mathcal{B}_{r}(t)\right)^{J}} \sup _{\bar{\alpha} \in \mathcal{A}_{r}(t)} \max _{i \in\{1, \ldots, I\}}\left\{\hat{p}_{i}-J_{i}^{q}(\bar{\alpha}, \hat{\beta})\right\}
$$


(the difference with (3.5) is that player I here can use random strategies.)

Let's denote by $e=e(\hat{p}, q)$ the right hand term of (3.6). First we prove that $e$ is convex with respect to $\hat{p}$ :

Fix $q \in \Delta(J), \hat{p}, \hat{p}^{\prime} \in \mathbb{R}^{I}$ and $a \in(0,1)$.

For $\epsilon>0$, let $\hat{\beta}$ (resp. $\left.\hat{\beta}^{\prime}\right) \in\left(\mathcal{B}_{r}(t)\right)^{J}$ be some $\epsilon$-optimal strategy for $e(\hat{p}, q)$ (resp. $e\left(\hat{p}^{\prime}, q\right)$ ). Set $\hat{p}^{a}=a \hat{p}+(1-a) \hat{p}^{\prime}$.

We define a new strategy $\hat{\beta}^{a} \in\left(\mathcal{B}_{r}(t)\right)^{J}$ by

$$
\bar{\beta}_{j}^{a}=\left(\beta_{j}^{1}, \ldots, \beta_{j}^{S}, \beta_{j}^{\prime 1}, \ldots, \beta_{j}^{\prime S^{\prime}} ;\left(s_{j}^{a}\right)^{1}, \ldots,\left(s_{j}^{a}\right)^{S+S^{\prime}}\right), \quad j \in\{1, \ldots, J\},
$$

with

$$
\left(s^{a}\right)_{j}^{k}= \begin{cases}a s_{j}^{k} & \text { for } k \in\{1, \ldots, S\}, \\ (1-a) s_{j}^{\prime k-S} & k \in\left\{S+1, \ldots, S+S^{\prime}\right\} .\end{cases}
$$

Let $\bar{\alpha} \in \mathcal{A}_{r}(t)$. Since the application $\left(x_{1}, \ldots, x_{I}\right) \rightarrow \max \left\{x_{i}, i=1, \ldots, I\right\}$ is convex, we have

$$
\begin{aligned}
\max _{i}\left\{\hat{p}_{i}^{a}-J_{i}^{q}\left(\bar{\alpha}, \hat{\beta}^{a}\right)\right\}= & \max _{i}\left\{a\left(\hat{p}_{i}-J_{i}^{q}(\bar{\alpha}, \hat{\beta})\right)+(1-a)\left(\hat{p}_{i}^{\prime}-J_{i}^{q^{\prime}}\left(\bar{\alpha}, \hat{\beta}^{\prime}\right)\right)\right\} \\
\leq & \operatorname{asup}_{\bar{\alpha} \in \mathcal{A}_{r}(t)} \max _{i}\left(\hat{p}_{i}-J_{i}^{q}\left(\bar{\alpha}, \hat{\beta}^{a}\right)\right) \\
& +(1-a) \sup _{\bar{\alpha} \in \mathcal{A}_{r}(t)} \max _{i}\left(\hat{p}_{i}^{1}-J_{i}^{q^{\prime}}\left(\bar{\alpha}, \hat{\beta}^{\prime}\right)\right) \\
\leq & a e(\hat{p}, q)+(1-a) e\left(\hat{p}^{\prime}, q\right)+\epsilon .
\end{aligned}
$$

Since $\epsilon$ is arbitrary, we can deduce that $e$ is convex with respect to $\hat{p}$.

The next step is to prove that $e^{*}=V^{-}$. By the convexity of $e$, this will imply that $V^{-*}=e$. We can reorganize $e^{*}(p, q)$ as follows :

$$
\begin{aligned}
e^{*}(p, q) & =\sup _{\hat{p} \in \mathbb{R}^{I}}\left\{\sum_{i=1}^{I} \hat{p}_{i} p_{i}+\sup _{\hat{\beta} \in\left(\mathcal{B}_{r}(t)\right)^{J}} \inf _{\bar{\alpha} \in \mathcal{A}_{r}(t)} \min _{i^{\prime} \in\{1, \ldots, I\}}\left\{J_{i^{\prime}}^{q}(\bar{\alpha}, \hat{\beta})-\hat{p}_{i^{\prime}}\right\}\right\} \\
& =\sup _{\hat{\beta} \in\left(\mathcal{B}_{r}(t)\right)^{J}} \sup _{\hat{p} \in \mathbb{R}^{I}} \sum_{i=1}^{I} p_{i} \min _{i^{\prime} \in\{1, \ldots, I\}}\left\{\inf _{\bar{\alpha} \in \mathcal{A}_{r}(t)} J_{i^{\prime}}^{q}(\bar{\alpha}, \hat{\beta})+\left(\hat{p}_{i}-\hat{p}_{i^{\prime}}\right)\right\}
\end{aligned}
$$

The supremum over $\hat{p} \in \mathbb{R}^{I}$ is attained for $\hat{p}_{i^{\prime}}=\inf _{\bar{\alpha} \in \mathcal{A}_{r}(t)} J_{i^{\prime}}^{q}(\bar{\alpha}, \hat{\beta})$ and we get the claimed result.

Finally, to get (3.5), it remains to show that player I can use non random strategies. Indeed, writing $V^{-*}$ as in (3.6) and since $\mathcal{A}(t) \subset \mathcal{A}_{r}(t)$, it is obvious that the left hand side 
of (3.5) is not smaller than the right hand side.

Concerning the reverse inequality, we can write

$$
\begin{aligned}
& \sup _{\bar{\alpha} \in \mathcal{A}_{r}(t)} \quad \max _{i}\left\{\hat{p}_{i}-J_{i}^{q}(\bar{\alpha}, \bar{\beta})\right\} \\
& \quad \leq \sup _{R \in \mathbb{N}^{*} \sup _{\left(\alpha^{1}, \ldots, \alpha^{R}\right) \in(\mathcal{A}(t))^{R},\left(r^{1}, \ldots, r^{R}\right) \in \Delta(R)} \sum_{k=1}^{R} r^{k} \max _{i}\left\{\hat{p}_{i}-J_{i}^{q}\left(\alpha^{k}, \hat{\beta}\right)\right\}} \\
& \leq \sup _{R \in \mathbb{N}^{*}} \sup _{\left(r^{1}, \ldots, r^{R}\right) \in \Delta(R)} \sum_{k} r^{k} \sup _{\alpha \in \mathcal{A}(t)} \max _{i}\left\{\hat{p}_{i}-J_{i}^{q}(\alpha, \hat{\beta})\right\} .
\end{aligned}
$$

The result follows after one recalls that $\sum_{k=1}^{R} r^{k}=1$.

Proposition 3.1 (Subdynamic programming for $V^{-*}$ )

For all $0 \leq t_{0} \leq t_{1} \leq T, x_{0} \in \mathbb{R}^{n}, \hat{p} \in \mathbb{R}^{I}, q \in \Delta(J)$, it holds that

$$
V^{-*}\left(t_{0}, x_{0}, \hat{p}, q\right) \leq \inf _{\beta \in \mathcal{B}\left(t_{0}\right)} \sup _{\alpha \in \mathcal{A}\left(t_{0}\right)} E\left[V^{-*}\left(t_{1}, X_{t_{1}}^{t_{0}, x_{0}, \alpha, \beta}, \hat{p}, q\right)\right]
$$

Proof : Set $V_{1}^{-*}\left(t_{0}, t_{1}, x_{0}, \hat{p}, q\right)=\inf _{\beta \in \mathcal{B}\left(t_{0}\right)} \sup _{\alpha \in \mathcal{A}\left(t_{0}\right)} E\left[V^{-*}\left(t_{1}, X_{t_{1}}^{t_{0}, x_{0}, \alpha, \beta}, \hat{p}, q\right)\right]$.

For $\epsilon>0$, let $\beta^{\epsilon} \in \mathcal{B}\left(t_{0}\right)$ be $\epsilon$-optimal for $V_{1}^{-*}\left(t_{0}, t_{1}, x_{0}, \hat{p}, q\right)$, and, for all $x \in \mathbb{R}^{n}$, let $\hat{\beta}^{x} \in\left(\mathcal{B}_{r}\left(t_{1}\right)\right)^{J}$ be $\epsilon$-optimal for $V^{-*}\left(t_{1}, x, \hat{p}, q\right)$. By the uniformly Lipschitz assumptions for the parameters of the dynamics, there exists $R>0$ such that, for all $\alpha \in \mathcal{A}\left(t_{0}\right)$,

$$
P\left[X_{t_{1}}^{t_{0}, x_{0}, \alpha, \beta^{\epsilon}} \in B\left(x_{0}, R\right)\right] \geq 1-\epsilon
$$

where $B\left(x_{0}, R\right)$ denotes the ball in $\mathbb{R}^{n}$ of center $x_{0}$ and radius $R$.

Remark that $J_{i}^{q}$ and $V^{-*}$ are uniformly Lipschitz continuous in $x$. This implies that we can find $r>0$ such that, for any $x \in \mathbb{R}^{n}$ and $y \in \mathcal{B}(x, r), \hat{\beta}^{x}$ is $2 \epsilon$-optimal for $V^{-*}\left(t_{1}, y, \hat{p}, q\right)$. Now let $x_{1}, \ldots, x_{M} \in \mathbb{R}^{n}$ such that $\cup_{m=1}^{M} B\left(x_{m}, \frac{r}{2}\right) \supset B\left(x_{0}, R\right)$.

Set $\hat{\beta}^{m}=\hat{\beta}^{x_{m}}$ for $m=1, \ldots, M$ and choose some arbitrary $\hat{\beta}^{0} \in\left(\mathcal{B}_{r}\left(t_{1}\right)\right)^{J}$.

Each $\hat{\beta}^{m}$ is detailed in the following way:

$$
\hat{\beta}^{m}=\left(\bar{\beta}_{1}^{m}, \ldots, \bar{\beta}_{J}^{m}\right),
$$

with

$$
\bar{\beta}_{j}^{m}=\left(\beta_{j}^{m, 1}, \ldots, \beta_{j}^{m, S_{j}^{m}} ; s_{j}^{m, 1}, \ldots, s_{j}^{m, S_{j}^{m}}\right) .
$$


Let $\delta$ be a common delay for $\hat{\beta}^{0}, \ldots, \hat{\beta}^{M}$ that we can choose as small as we need : $0<\delta<\frac{r^{2} \epsilon}{4 C} \wedge\left(t_{1}-t_{0}\right)$, where $C>0$ is defined through the parameters of the dynamics by

$$
\forall \alpha \in \mathcal{A}(t), \beta \in \mathcal{B}(t), t, t^{\prime} \in\left[t_{0}, T\right], E\left[\left|X_{t}^{t_{0}, x_{0}, \alpha, \beta}-X_{t^{\prime}}^{t_{0}, x_{0}, \alpha, \beta}\right|^{2}\right] \leq C\left|t-t^{\prime}\right| .
$$

We then have in particular, for all $\alpha \in \mathcal{A}(t)$ and $\beta \in \mathcal{B}(t)$,

$$
P\left[\left|X_{t_{1}}^{t_{0}, x_{0}, \alpha, \beta}-X_{t_{1}-\delta}^{t_{0}, x_{0}, \alpha, \beta}\right|>\frac{r}{2}\right] \leq \epsilon .
$$

Let $\left(E_{m}\right)_{m=1, \ldots, M}$ be a Borel measurable partition of $B\left(x_{0}, R\right)$, such that, for all $m \in$ $\{1, \ldots, M\}, E_{m} \subset B\left(x_{m}, \frac{r}{2}\right)$. Set $E_{0}=B\left(x_{0}, R\right)^{c}$.

We are now able to define a new strategy for player II, $\hat{\beta}^{\epsilon} \in\left(\mathcal{B}_{r}\left(t_{0}\right)\right)^{J}$ :

Fix $j \in\{1, \ldots, J\}$. For $l=\left(l_{0}, \ldots, l_{M}\right) \in L:=\Pi_{m=0}^{M}\left\{1, \ldots, S_{j}^{m}\right\}$, set $s_{j}^{l}=\Pi_{m=0}^{M} s_{j}^{m, l_{m}}$. Remark that $\left\{s_{j}^{l}, l \in L\right\} \in \Delta(\operatorname{Card}(L))$.

Then, for $l \in L, l=\left(l_{0}, \ldots, l_{M}\right)$, we define $\left(\beta_{j}^{\epsilon}\right)^{l} \in \mathcal{B}\left(t_{0}\right)$ by

$$
\forall f \in C\left(\left[t_{0}, T\right], \mathbb{R}^{n}\right), \forall t \in\left[t_{0}, T\right]
$$

$$
\left(\beta_{j}^{\epsilon}\right)^{l}(t, f)= \begin{cases}\beta^{\epsilon}(t, f) & \text { if } t \in\left[t_{0}, t_{1}\right), \\ \beta_{j}^{m, l_{m}}\left(t,\left.f\right|_{\left[t_{1}, T\right]}\right) & \text { if } t \in\left[t_{1}, T\right] \text { and } f\left(t_{1}-\delta\right) \in E_{m} .\end{cases}
$$

We set $\bar{\beta}_{j}^{\epsilon}:=\left(\left(\beta_{j}^{\epsilon}\right)^{l} ; s_{j}^{l}, l \in L\right) \in \mathcal{B}_{r}\left(t_{0}\right)$, and finally $\hat{\beta}^{\epsilon}=\left(\bar{\beta}_{1}^{\epsilon}, \ldots, \bar{\beta}_{J}^{\epsilon}\right)$.

For some fixed $\alpha \in \mathcal{A}\left(t_{0}\right)$ and $f \in C\left(\left[t_{0}, t_{1}\right], \mathbb{R}^{n}\right)$, we define a new strategy $\alpha_{f} \in \mathcal{A}\left(t_{1}\right)$ by: for all $t \in[0, T]$ and $f^{\prime} \in C\left(\left[t_{1}, T\right], \mathbb{R}^{n}\right)$,

$$
\alpha_{f}\left(t, f^{\prime}\right)=\alpha(t, \tilde{f}), \text { with } \tilde{f}(t)=\left\{\begin{array}{l}
f(t) \text { for } t \in\left[t_{0}, t_{1}\right], \\
f^{\prime}(t)-f^{\prime}\left(t_{1}\right)+f\left(t_{1}\right), \text { for } t \in\left(t_{1}, T\right] .
\end{array}\right.
$$

Set $X^{\epsilon} .=X^{t_{0}, x_{0}, \alpha, \beta^{\epsilon}}$ and, for $m \in\{0, \ldots, M\}, A_{m}=\left\{X_{t_{1}-\delta}^{\epsilon} \in E_{m}\right\}$. Set further $A=\left\{\left|X_{t_{1}}^{\epsilon}-X_{t_{1}-\delta}^{\epsilon}\right| \leq \frac{r}{2}\right\}$. By (3.7), it holds that $P\left[A^{c}\right] \leq \epsilon$. Remark also that, on each $A \cap A_{m}, X_{t_{1}}^{\epsilon}$ belongs to $B\left(x_{m}, r\right)$ and consequently, still on $A \cap A_{m}, \hat{\beta}^{m}$ is $2 \epsilon$-optimal for $V^{-*}\left(t_{1}, X_{t_{1}}^{\epsilon}, \hat{p}, q\right)$.

For all $i \in\{1, \ldots, I\}, j \in\{1, \ldots, J\}$ and $l \in L$, we have

$$
E\left[g_{i j}\left(X_{T}^{t_{0}, x_{0}, \alpha,\left(\beta_{j}^{\epsilon}\right)^{l}}\right) \mid \mathcal{F}_{t_{1}}\right]=\left.\sum_{m=0}^{M} 1_{A_{m}} E\left[g_{i j}\left(X_{T}^{t_{1}, y, \alpha_{f}, \beta_{j}^{m, l_{m}}}\right)\right]\right|_{y=X_{t_{1}}^{\epsilon}, f=X_{.}^{\epsilon} \mid\left[t_{0}, t_{1}\right]} .
$$

It follows that

$$
\begin{aligned}
J_{i}^{q}\left(t_{0}, x_{0}, \alpha, \hat{\beta}^{\epsilon}\right) & =\sum_{j=1}^{J} q_{j} \sum_{l \in L} s_{j}^{l} E\left[g_{i j}\left(X_{T}^{t_{0}, x_{0}, \alpha,\left(\beta_{j}^{\epsilon}\right)^{l}}\right)\right] \\
& =E\left[\sum _ { m = 0 } ^ { M } 1 _ { A _ { m } } J _ { i } ^ { q } \left(t_{1}, X_{t_{1}}^{\epsilon}, \alpha_{\left.\left.\left.X^{\epsilon}\right|_{\left[t_{0}, t_{1}\right]}, \hat{\beta}^{m}\right)\right]}\right.\right.
\end{aligned}
$$


And

$$
\begin{aligned}
\max _{i \in\{1, \ldots, I\}}\left\{\hat{p}_{i}-\right. & \left.J_{i}^{q}\left(t_{0}, x_{0}, \alpha, \hat{\beta}^{\epsilon}\right)\right\} \\
\leq & E\left[\sum _ { m = 0 } ^ { M } { } ^ { 1 } A _ { m } \operatorname { m a x } _ { i \in \{ 1 , \ldots , I \} } \left\{\hat{p}_{i}-J_{i}^{q}\left(t_{1}, X_{t_{1}}^{\epsilon}, \alpha_{\left.\left.\left.\left.X^{\epsilon}\right|_{\left[t_{0}, t_{1}\right]}, \hat{\beta}^{m}\right)\right\}\right]}\right.\right.\right. \\
\leq & E\left[\sum_{m=0}^{M}{ }^{1} A_{m}\left(\sup _{\alpha \in \mathcal{A}\left(t_{1}\right)} \max _{i \in\{1, \ldots, I\}}\left\{\hat{p}_{i}-J_{i}^{q}\left(t_{1}, X_{t_{1}}^{\epsilon}, \alpha, \hat{\beta}^{m}\right)\right\}\right)\right] \\
\leq & E\left[\left(V^{-*}\left(t_{1}, X_{t_{1}}^{\epsilon}, \hat{p}, q\right)+2 \epsilon\right) 1_{A \cap\left\{X_{t_{1}}^{\epsilon} \in B\left(x_{0}, R\right)\right\}}\right] \\
& \quad+\max _{i \in\{1, \ldots, I\}}\left\{\left|\hat{p}_{i}\right|+K\right\}\left(P\left[A^{c}\right]+P\left[X_{t_{1}}^{\epsilon} \notin B\left(x_{0}, R\right)\right]\right),
\end{aligned}
$$

by the choice of $\left(\hat{\beta}^{m}, m \in\{1, \ldots, M\}\right)$ and where $K$ is an upper bound of $|g|$.

By the choice of $R$ and with the notation $K(\hat{p})=4 \max _{i \in\{1, \ldots, I\}}\left\{\left|\hat{p}_{i}\right|+K\right\}+\epsilon$, we get

$$
\begin{aligned}
\max _{i \in\{1, \ldots, I\}}\left\{\hat{p}_{i}-J_{i}^{q}\left(t_{0}, x_{0}, \alpha, \hat{\beta}^{\epsilon}\right)\right\} & \leq E\left[V^{-*}\left(t_{1}, X_{t_{1}}^{\epsilon}, \hat{p}, q\right)+2 \epsilon\right]+K(\hat{p}) \epsilon \\
& \leq \sup _{\alpha \in \mathcal{A}\left(t_{0}\right)} E\left[V^{-*}\left(t_{1}, X_{t_{1}}^{t_{0}, x_{0}, \alpha, \beta^{\epsilon}}, \hat{p}, q\right)\right]+2 \epsilon(1+K(\hat{p})) \\
& \leq V_{1}^{-*}\left(t_{0}, t_{1}, x_{0}, \hat{p}, q\right)+\epsilon(3+2 K(\hat{p}))
\end{aligned}
$$

(for the last inequality, recall that $\beta^{\epsilon}$ was chosen $\epsilon$-optimal for $V_{1}^{-*}\left(t_{0}, t_{1}, x_{0}, \hat{p}, q\right)$ ).

We can deduce the result.

A classical consequence of the subdynamic programming principle for $V^{-*}$ is that this function is a subsolution of some associated Hamilton-Jacobi equation. We give a proof of that result for sake of completeness.

Corollary 3.1 For any $(\hat{p}, q) \in \mathbb{R}^{I} \times \Delta(J), V^{-*}(\cdot, \cdot, \hat{p}, q)$ is a subsolution in the viscosity sense of

$$
w_{t}+H^{-*}\left(t, x, D w, D^{2} w\right)=0, \quad(t, x) \in(0, T) \times \mathbb{R}^{n}
$$

with

$$
\begin{aligned}
& H^{-*}(t, x, p, A)=-H^{-}(t, x,-p,-A)= \\
& \quad \inf _{v \in V} \sup _{u \in U}\left\{\langle b(t, x, u, v), p\rangle+\frac{1}{2} \operatorname{Tr}\left(A \sigma(t, x, u, v) \sigma^{*}(t, x, u, v)\right)\right\} .
\end{aligned}
$$

Proof : For $\left(t_{0}, x_{0}\right) \in[0, T] \times \mathbb{R}^{n}, \hat{p} \in \mathbb{R}^{I}, q \in \Delta(J)$ fixed, let $\phi \in C^{1,2}$ such that $\phi\left(t_{0}, x_{0}\right)=$ $V^{-*}\left(t_{0}, x_{0}, \hat{p}, q\right)$ and, for all $(s, y) \in[0, T] \times \mathbb{R}^{n}, \phi(s, y) \geq V^{-*}(s, y, \hat{p}, q)$.

We have to prove that

$$
\phi_{t}\left(t_{0}, x_{0}\right)+H^{-*}\left(t_{0}, x_{0}, D \phi\left(t_{0}, x_{0}\right), D^{2}\left(t_{0}, x_{0}\right)\right) \geq 0
$$


Suppose that this is false and consider $\theta>0$ such that

$$
\phi_{t}\left(t_{0}, x_{0}\right)+H^{-*}\left(t_{0}, x_{0}, D \phi\left(t_{0}, x_{0}\right), D^{2}\left(t_{0}, x_{0}\right)\right) \leq-\theta<0 \text {. }
$$

Set $\Lambda(t, x, u, v)=\phi_{t}(t, x)+\langle b(t, x, u, v), D \phi(t, x)\rangle+\operatorname{Tr}\left(D^{2} \phi(t, x) \sigma(t, x, u, v) \sigma^{*}(t, x, u, v)\right)$. Since, for fixed $\hat{p}, V^{-*}$ is bounded, we can choose $\phi$ such that $\phi_{t}$ and $D^{2} \phi$ are also bounded. It follows that, for some $K>0$, we have $|\Lambda(t, x, u, v)| \leq K$.

Now the relation (3.9) is equivalent to

$$
\inf _{v \in V} \sup _{u \in U} \Lambda\left(t_{0}, x_{0}, u, v\right) \leq-\theta .
$$

This implies the existence of a control $v_{0} \in V$ such that, for all $u \in U$,

$$
\Lambda\left(t_{0}, x_{0}, u, v_{0}\right) \leq-\frac{2 \theta}{3} .
$$

Moreover, since $\Lambda$ is continuous in $(t, x)$, uniformly in $u, v$, we can find $R>0$ such that,

$$
\forall(t, x) \in\left[t_{0}, T\right] \times \mathbb{R}^{n},\left|t-t_{0}\right| \vee\left\|x-x_{0}\right\|<R, \forall u \in U, \Lambda\left(t, x, u, v_{0}\right) \leq-\frac{\theta}{2} .
$$

Now define a strategy for player II by $\beta_{0}(t, f)=v_{0}$ for all $(t, f) \in\left[t_{0}, T\right] \times C\left(\left[t_{0}, T\right], \mathbb{R}^{n}\right)$.

Fix $\epsilon>0$ and $t \in\left(t_{0}, R\right)$. Because of the subdynamical programming (Proposition 3.1), there exists $\alpha_{\epsilon, t} \in \mathcal{A}\left(t_{0}\right)$ such that

$$
E\left[V^{-*}\left(t_{1}, X_{t_{1}}^{t_{0}, x_{0}, \alpha_{\epsilon}, t, \beta_{0}}, \hat{p}, q\right)\right]-V^{-*}\left(t_{0}, x_{0}, \hat{p}, q\right) \geq-\epsilon\left(t-t_{0}\right) .
$$

Let $\left(u_{s}, v_{s}\right) \in \mathcal{U}\left(t_{0}\right) \times \mathcal{V}\left(t_{0}\right)$ the controls associated to $\left(\alpha_{\epsilon, t}, \beta_{0}\right)$ by the relation (2.3) and set $X$. $=X^{t_{0}, x_{0}, \alpha_{\epsilon, t}, \beta_{0}}=X^{t_{0}, x_{0}, u, v}$. (Remark that, by the choice of $\beta_{0},\left(v_{s}\right)$ is constant and equal to $v_{0}$.)

Now we write Itô's formula for $\phi\left(t, X_{t}\right)$ :

$$
\begin{aligned}
\phi\left(t, X_{t}\right)-\phi\left(t_{0}, x_{0}\right)= & \int_{t_{0}}^{t} \Lambda\left(s, X_{s}, u_{s}, v_{s}\right) d s \\
& +\int_{t_{0}}^{t}\left\langle D \phi\left(s, X_{s}\right), b\left(s, X_{s}, u_{s}, v_{s}\right)\right\rangle d B_{s} .
\end{aligned}
$$

By (3.11), (3.12) and the definition of $\phi$, we have

$$
E\left[\int_{t_{0}}^{t} \Lambda\left(s, X_{s}, u_{s}, v_{s}\right) d s\right] \geq-\epsilon\left(t-t_{0}\right)
$$

In the other hand, there exists a constant $C>0$ depending only on the parameters of $X$, such that

$$
P\left[\left\|X .-x_{0}\right\|_{t}>R\right] \leq \frac{C\left(t-t_{0}\right)^{2}}{R^{4}},
$$


with the notation $\|f\|_{t}=\sup _{s \in\left[t_{0}, t\right]}\|f(s)\|$.

Following (3.10), this implies that, for all $t \in\left[t_{0}, T \wedge\left(t_{0}+R\right)\right]$,

$$
E\left[I_{\left\{\left\|X .-x_{0}\right\|_{t}<R\right\}} \int_{t_{0}}^{t} \Lambda\left(s, X_{s}, u_{s}, v_{s}\right) d s\right] \leq-\frac{\theta}{2}\left(t-t_{0}\right) .
$$

By (3.13) and (3.14), we now have

$$
\begin{aligned}
-\epsilon\left(t-t_{0}\right) & \leq E\left[\int_{t_{0}}^{t} \Lambda\left(s, X_{s}, u_{s}, v_{s}\right) d s I_{\left\{\left\|X .-x_{0}\right\|_{t}>R\right\}}\right]+E\left[\int_{t_{0}}^{t} \Lambda\left(s, X_{s}, u_{s}, v_{s}\right) d s I_{\left\{\left\|X .-x_{0}\right\|_{t} \leq R\right\}}\right] \\
& \leq \frac{K C}{R^{4}}\left(t-t_{0}\right)^{2}-\frac{\theta}{2}\left(t-t_{0}\right)
\end{aligned}
$$

or, equivalently,

$$
\frac{\theta}{2} \leq \frac{K C}{R^{4}}\left(t-t_{0}\right)+\epsilon
$$

Since $t-t_{0}$ and $\epsilon$ can be chosen arbitrarily small, we get a contradiction.

For $V^{+}$we have:

Proposition 3.2 (Superdynamic programming and HJI equation for $V^{+\sharp}$ )

For all $0 \leq t_{0} \leq t_{1} \leq T, x_{0} \in \mathbb{R}^{n}, p \in \Delta(I), \hat{q} \in \mathbb{R}^{J}$, it holds that

$$
V^{+\sharp}\left(t_{0}, x_{0}, p, \hat{q}\right) \geq \inf _{\beta \in \mathcal{B}\left(t_{0}\right)} \sup _{\alpha \in \mathcal{A}\left(t_{0}\right)} E\left[V^{+\sharp}\left(t_{1}, X_{t_{1}}^{t_{0}, x_{0}, \alpha, \beta}, p, \hat{q}\right)\right] .
$$

As a consequence, for any $(p, \hat{q}) \in \Delta(I) \times \mathbb{R}^{J}, V^{+\sharp}(\cdot, \cdot, p, \hat{q})$ is a supersolution in viscosity sense of

$$
\left.w_{t}+H^{+*}\left(t, x, D w, D^{2} w\right)\right)=0, \quad(t, x) \in(0, T) \times \mathbb{R}^{n},
$$

where

$$
\begin{aligned}
& H^{+*}(t, x, p, A)=-H^{+}(t, x,-p,-A)= \\
& \quad \sup _{u \in U} \inf _{v \in V}\left\{\langle b(t, x, u, v), p\rangle+\frac{1}{2} \operatorname{Tr}\left(A \sigma(t, x, u, v) \sigma^{*}(t, x, u, v)\right)\right\} .
\end{aligned}
$$

Proof : We note that $V^{+}$is equal to the opposite of the lower value of the game in which we replace $g_{i j}$ by $-g_{i j}$, Player I is the maximizer and in which the respective roles of $p$ and $q$ are exchanged. Using Proposition 3.1 in this framework gives the superdynamic programming principle. Now Corollary 3.1 shows that, for any $(p, \hat{q}) \in \Delta(I) \times \mathbb{R}^{J}$, $\left(-V^{+}\right)^{*}(\cdot, \cdot, p, \hat{q})=-V^{+\sharp}(\cdot, \cdot, p,-\hat{q})$ is a subsolution of

$$
\left.w_{t}+H^{+}\left(t, x, D w, D^{2} w\right)\right)=0, \quad(t, x) \in(0, T) \times \mathbb{R}^{n} .
$$


Hence $V^{+\sharp}(\cdot, \cdot, p,-\hat{q})$ is a supersolution of

$$
\left.w_{t}+H^{+*}\left(t, x, D w, D^{2} w\right)\right)=0, \quad(t, x) \in(0, T) \times \mathbb{R}^{n} .
$$

Since this holds true for any $(p, \hat{q})$, this proves our claim.

\section{Comparison principle and existence of a value}

In this section we first state a new comparison principle and apply it to get the existence and the characterization of the value. Then we give a proof for the comparison principle.

\subsection{Statement of the comparison principle and existence of a value}

Let $H:[0, T] \times \mathbb{R}^{n} \times \mathbb{R}^{n} \times \mathcal{S}_{n} \times \Delta(I) \times \Delta(J) \rightarrow \mathbb{R}$ be continuous and satisfy

$$
\begin{aligned}
& H\left(s, y, \xi_{2}, X_{2}, p, q\right)-H\left(t, x, \xi_{1}, X_{1}, p, q\right) \geq \\
& \quad-\omega\left(\left|\xi_{1}-\xi_{2}\right|+a|(t, x)-(s, y)|^{2}+b+|(t, x)-(s, y)|\left(1+\left|\xi_{1}\right|+\left|\xi_{2}\right|\right)\right)
\end{aligned}
$$

where $\omega$ is continuous and non decreasing with $\omega(0)=0$, for any $a, b \geq 0,(p, q) \in \Delta(I) \times$ $\Delta(J), s, t \in[0, T], x, y, \xi_{1}, x_{2} \in \mathbb{R}^{n}$ and $X_{1}, X_{2} \in \mathcal{S}_{n}$ such that

$$
\left(\begin{array}{ll}
-X_{1} & 0 \\
0 & X_{2}
\end{array}\right) \leq a\left(\begin{array}{cc}
I & -I \\
-I & I
\end{array}\right)+b I
$$

Definition 4.1 We say that a map $w:(0, T) \times \mathbb{R}^{n} \times \Delta(I) \times \Delta(J) \rightarrow \mathbb{R}$ is a supersolution in the dual sense of equation

$$
w_{t}+H\left(t, x, D w, D^{2} w, p, q\right)=0
$$

if $w=w(t, x, p, q)$ is lower semicontinuous, concave with respect to $q$ and if, for any $\mathcal{C}^{2}\left((0, T) \times \mathbb{R}^{n}\right)$ function $\phi$ such that $(t, x) \rightarrow w^{*}(t, x, \hat{p}, \bar{q})-\phi(t, x)$ has a maximum at some point $(\bar{t}, \bar{x})$ for some $(\hat{p}, \bar{q}) \in \mathbb{R}^{I} \times \Delta(J)$, we have

$$
\phi_{t}(\bar{t}, \bar{x})-H\left(\bar{t}, \bar{x},-D \phi(\bar{t}, \bar{x}),-D^{2} \phi(\bar{t}, \bar{x}), p, \bar{q}\right) \geq 0 \quad \forall p \in \partial_{\hat{p}}^{-} w^{*}(\bar{t}, \bar{x}, \hat{p}, \bar{q}) .
$$

We say that $w$ is a subsolution of (4.17) in the dual sense if $w$ is upper semicontinuous, convex with respect to $p$ and if, for any $\mathcal{C}^{2}\left((0, T) \times \mathbb{R}^{n}\right)$ function $\phi$ such that $(t, x) \rightarrow$ $w^{\sharp}(t, x, \bar{p}, \hat{q})-\phi(t, x)$ has a minimum at some point $(\bar{t}, \bar{x})$ for some $(\bar{p}, \hat{q}) \in \Delta(I) \times \mathbb{R}^{J}$, we have

$$
\phi_{t}(\bar{t}, \bar{x})-H\left(\bar{t}, \bar{x},-D \phi(\bar{t}, \bar{x}),-D^{2} \phi(\bar{t}, \bar{x}), \bar{p}, q\right) \leq 0 \quad \forall q \in \partial_{\hat{q}}^{+} w^{\sharp}(\bar{t}, \bar{x}, \bar{p}, \hat{q})
$$


A solution of (4.17) in the dual sense is a map which is sub-and supersolution in the dual sense.

\section{Remarks :}

1. We have proved in Corollary 3.1 that $V^{-}$is a dual supersolution of the HJ equation

$$
w_{t}+H^{-}\left(t, x, D w, D^{2} w\right)=0
$$

where $H^{-}$is defined by (3.8), while Proposition 3.2 shows that $V^{+}$is a dual subsolution of the HJ equation

$$
w_{t}+H^{+}\left(t, x, D w, D^{2} w\right)=0,
$$

where $H^{-}$is defined by (3.15).

2. The necessity to deal with a Hamiltonian $H$ with a $(p, q)$ dependence will become clear in the next section where we study differential games with running costs.

3. An equivalent definition of the notion of dual super- or subsolution in given in Lemma 5.3 below.

The main result of this section is the following:

Theorem 4.1 (Comparison principle) Let us assume that $H$ satisfies the structure condition (4.16). Let $w_{1}$ be a bounded, Hölder continuous subsolution of (4.17) in the dual sense which is uniformly Lipschitz continuous w.r. to $q$ and $w_{2}$ be a bounded, Hölder continuous supersolution of (4.17) in the dual sense which is uniformly Lipschitz continuous w.r. to p. Assume that

$$
w_{1}(T, x, p, q) \leq w_{2}(T, x, p, q) \quad \forall(x, p, q) \in \mathbb{R}^{n} \times \Delta(I) \times \Delta(J) .
$$

Then

$$
w_{1}(t, x, p, q) \leq w_{2}(t, x, p, q) \quad \forall(t, x, p, q) \in[0, T] \times \mathbb{R}^{n} \times \Delta(I) \times \Delta(J) .
$$

Remark : For simplicity we are assuming here that $w_{1}$ and $w_{2}$ are Hölder continuous and bounded. These assumptions could be relaxed by standard (but painfull) techniques. We do not know if the uniform Lipschitz continuity assumption on $w_{1}$ with respect to $q$ and on $w_{2}$ with respect to $p$ can be relaxed.

As a consequence we have 
Theorem 4.2 (Existence of a value) Under assumptions (H), 2.4) and (2.2), the game has a value:

$$
V^{+}(t, x, p, q)=V^{-}(t, x, p, q) \quad \forall(t, x, p, q) \in(0, T) \times \mathbb{R}^{n} \times \Delta(I) \times \Delta(J) .
$$

Furthermore $V^{+}=V^{-}$is the unique solution in the dual sense of HJI equation (4.17) with terminal condition

$$
V^{+}(T, x, p, q)=V^{-}(T, x, p, q)=\sum_{i=1}^{I} \sum_{j=1}^{J} p_{i} q_{j} g_{i j}(x) \quad \forall(x, p, q) \in \mathbb{R}^{n} \times \Delta(I) \times \Delta(J) .
$$

Proof of Theorem 4.2 : The Hamiltonian H defined by (2.2) is known to satisfy (4.16) (see [9] for instance). ¿From the definition of $V^{+}$and $V^{-}$we have $V^{-} \leq V^{+}$. We have proved in Lemma 2.2 and Proposition 2.1 that $V^{+}$and $V^{-}$are Hölder continuous, Lipschitz continuous with respect to $p$ and $q$, convex w.r. to $p$ and concave w.r. to $q$. From Corollary 3.1 we know that $V^{-}$is a supersolution of (4.17) in the dual sense while Proposition 3.2 states that $V^{+}$is a supersolution of that same equation in the dual sense. The comparison principle then states that $V^{+} \leq V^{-}$, whence the existence and the characterization of the value: $V^{+}=V^{-}$is the unique solution in the dual sense of HJI equation (4.17).

\subsection{Proof of the comparison principle}

The proof of Theorem 4.1 relies on two arguments: first on a reformulation of the notions of sub- and supersolutions by using sub- and superjets; second on a new maximum principle described in the appendix.

Let us recall the notions of sub- and superjets of a function $w:(0, T) \times \mathbb{R}^{n} \rightarrow \mathbb{R}$ : the subjet $D^{2,-} w(\bar{t}, \bar{x})$ is the set of $\left(\xi_{t}, \xi_{x}, X\right) \in \mathbb{R}^{n+1} \times \mathcal{S}_{n}$ such that

$$
\left.w(t, x) \geq w(\bar{t}, \bar{x})+\xi_{t}(t-\bar{t})+\xi_{x} \cdot(x-\bar{x})+\frac{1}{2} X(x-\bar{x}) \cdot(x-\bar{x})+o\left(|t-\bar{t}|+|x-\bar{x}|^{2}\right)\right\}
$$

and the superjet $D^{2,+} w$ is given by

$$
D^{2,+} w(\bar{t}, \bar{x})=-D^{2,-}(-w)(\bar{t}, \bar{x})
$$

When $w$ depends on other variables $\left((p, q)\right.$ or $(p, \hat{q})$ for instance), $D^{2,-} w$ and $D^{2,+} w$ always denote the sub- and superjets with respect to the $(t, x)$ variables only. For $w=w(t, x, p, \hat{q})$, 
we set

$$
\overline{D^{2,-}} w(\bar{t}, \bar{x}, p, \hat{q})=\left\{\begin{array}{c}
\left(\xi_{t}, \xi_{x}, X\right) \in \mathbb{R}^{n+1} \times \mathcal{S}_{n}, \exists\left(t_{n}, x_{n}, p_{n}, \hat{q}_{n}\right) \rightarrow(\bar{t}, \bar{x}, p, \hat{q}), \\
\exists\left(\xi_{t}^{n}, \xi_{x}^{n}, X^{n}\right) \in D^{2,-} w\left(t_{n}, x_{n}, p_{n}, \hat{q}_{n}\right) \\
\text { with }\left(\xi_{t}^{n}, \xi_{x}^{n}, X^{n}\right) \rightarrow\left(\xi_{t}, \xi_{x}, X\right)
\end{array}\right\} .
$$

We use a symmetric notation for $\overline{D^{2,+}} w(\bar{t}, \bar{x}, \hat{p}, q)$.

The following equivalent formulation of the notion of sub- and supersolution is standard in viscosity solution theory, so we omit the proof:

Proposition 4.3 A map $w:(0, T) \times \mathbb{R}^{n} \times \Delta(I) \times \Delta(J) \rightarrow \mathbb{R}$ is a supersolution of equation (4.17) in the dual sense if and only if $w=w(t, x, p, q)$ is lower semicontinuous, concave with respect to $q$ and if, for any $(\bar{t}, \bar{x}, \hat{p}, \bar{q})$ and any $\left(\xi_{t}, \xi_{x}, X\right) \in \overline{D^{2,+}} w^{*}(\bar{t}, \bar{x}, \hat{p}, \bar{q})$ we have

$$
\xi_{t}-H\left(\bar{t}, \bar{x},-\xi_{x},-X, p, \bar{q}\right) \geq 0 \quad \forall p \in \partial_{\hat{p}}^{-} w^{*}(\bar{t}, \bar{x}, \hat{p}, \bar{q})
$$

Symmetrically $w$ is a subsolution of (4.17) in the dual sense if and only if $w$ is upper semicontinuous, convex with respect to $p$ and if, for any $(\bar{t}, \bar{x}, \bar{p}, \hat{q})$ and any $\left(\xi_{t}, \xi_{x}, X\right) \in$ $\overline{D^{2,-}} w^{\sharp}(\bar{t}, \bar{x}, \bar{p}, \hat{q})$ we have

$$
\xi_{t}-H\left(\bar{t}, \bar{x},-\xi_{x},-X, \bar{p}, q\right) \leq 0 \quad \forall q \in \partial_{\hat{q}}^{+} w^{\sharp}(\bar{t}, \bar{x}, \bar{p}, \hat{q})
$$

Proof of Theorem 4.1 : Let us assume that

$$
\sup _{t, x, p, q}\left(w_{1}-w_{2}\right)>0
$$

Since $w_{1}$ and $w_{2}$ are Hölder continuous and bounded, classical arguments show that

$$
M_{\epsilon, \eta, \alpha}:=\sup _{t, x, s, y, p, q}\left\{w_{1}(t, x, p, q)-w_{2}(s, y, p, q)-\left(\frac{|(t, x)-(s, y)|^{2}}{2 \epsilon}+\frac{\alpha}{2}\left(|x|^{2}+|y|^{2}\right)\right)+\eta t\right\}
$$

is finite and achieved at a point $\left(\bar{t}, \bar{x}, \bar{s}, \bar{y}, \bar{p}_{0}, \bar{q}_{0}\right)$. One can also show that

$$
\lim _{\epsilon, \eta, \alpha \rightarrow 0^{+}} M_{\epsilon, \eta, \alpha}=\sup _{t, x, p, q}\left(w_{1}-w_{2}\right)
$$

and that

$$
\frac{|(\bar{t}, \bar{x})-(\bar{s}, \bar{y})|^{2}}{\epsilon^{2}}, \alpha|\bar{x}|^{2}, \alpha|\bar{y}|^{2} \leq 2 M_{\infty}
$$

where $M_{\infty}=\left|w_{1}\right|_{\infty}+\left|w_{2}\right|_{\infty}$. Using (4.18) and the Hölder continuity of $w_{1}$ and $w_{2}$ shows that $\bar{t}<T$ and $\bar{s}<T$ as soon as $\epsilon, \eta$ and $\alpha$ are small enough. 
¿From the maximum principle (Theorem 6.1 stated in the Appendix), there are $(\bar{p}, \bar{q})$, $(\hat{p}, \hat{q})$ and $X_{1}, X_{2} \in \mathcal{S}_{n}$ such that

$$
\begin{gathered}
\bar{p} \in \partial_{\hat{p}}^{-} w_{2}^{*}(\bar{s}, \bar{y}, \hat{p}, \bar{q}), \bar{q} \in \partial_{\hat{q}}^{-} w_{2}^{\sharp}(\bar{t}, \bar{x}, \bar{p}, \hat{q}), \\
\left(-\frac{(\bar{t}-\bar{s})}{\epsilon}+\eta,-\frac{(\bar{x}-\bar{y})}{\epsilon}-\alpha \bar{x}, X_{1}\right) \in \overline{D^{2,-}} w_{1}^{\sharp}(\bar{s}, \bar{x}, \bar{p}, \hat{q}), \\
\left(\frac{(\bar{s}-\bar{t})}{\epsilon}, \frac{(\bar{y}-\bar{x})}{\epsilon}-\alpha \bar{y}, X_{2}\right) \in \overline{D^{2,+}} w_{2}^{*}(\bar{s}, \bar{y}, \hat{p}, \bar{q})
\end{gathered}
$$

and

$$
\left(\begin{array}{ll}
-X_{1} & 0 \\
0 & X_{2}
\end{array}\right) \leq\left(\frac{3}{\epsilon}+2 \alpha\right)\left(\begin{array}{cc}
I & -I \\
-I & I
\end{array}\right)+\left(\alpha+\alpha^{2} \epsilon\right) I
$$

Since $w_{1}$ is a subsolution of (4.17) in the dual sense and $\bar{q} \in \partial_{\hat{q}}^{-} w_{2}^{\sharp}(\bar{t}, \bar{x}, \bar{p}, \hat{q})$, Proposition 4.3 states that

$$
\eta-\frac{\bar{t}-\bar{s}}{\epsilon}-H\left(\bar{t}, \bar{x}, \frac{\bar{x}-\bar{y}}{\epsilon}+\alpha \bar{x},-X_{1}, \bar{p}, \bar{q}\right) \leq 0 .
$$

In the same way, since $w_{2}$ is a supersolution of (4.17) in the dual sense and $\bar{p} \in \partial_{\hat{p}}^{-} w_{2}^{*}(\bar{s}, \bar{y}, \hat{p}, \bar{q})$, we have

$$
\frac{(\bar{s}-\bar{t})}{\epsilon}-H\left(\bar{s}, \bar{y},-\frac{(\bar{y}-\bar{x})}{\epsilon}+\alpha \bar{y},-X_{2}, \bar{p}, \bar{q}\right) \geq 0,
$$

Using the structure condition (4.16) on $H$, and plugging estimates (4.19), (4.20) and (4.21) into (4.22) and (4.23) yields to a contradiction for $\epsilon, \alpha$ and $\eta$ sufficiently small as in [9].

\section{Games with running cost}

We now investigate differential games with asymmetric information on the running cost and on the terminal cost. The framework is basically the same as before. At the initial time, the cost (now consisting in a running cost and a terminal one) is chosen at random among $I \times J$ possible costs. The index $i$ is announced to Player I while the index $j$ is announced to Player II. Then the players play the game in order, for Player I to minimize the payoff and for Player II to maximize it.

In this section we keep the same terminology and the same notations as in the previous part. There is however a main difference: as we shall see later, in a game with a running cost, each player needs the knowledge of this running cost to build his strategy. Since we assume that the running cost depends on the control of both players, this means that 
the players have to observe the control of their opponent. This was not the case of the game before where the players only observed the state of the system. For this reason we have to change the notion of strategy: in this section the notion of strategies introduced in Definition 2.2 is replaced by the following one:

Definition 5.1 A strategy for player I starting at time $t$ is a Borel-measurable map $\alpha$ : $[t, T] \times \mathcal{C}\left([t, T], \mathbb{R}^{n}\right) \times L^{2}([t, T], V) \rightarrow U$ for which there exists $\delta>0$ such that, for all $s \in[t, T], f, f^{\prime} \in \mathcal{C}\left([t, T], \mathbb{R}^{n}\right)$ and $g, g^{\prime} \in L^{2}([t, T], V)$, if $f=f^{\prime}$ and $g=g^{\prime}$ a.e. on $[t, s]$, then $\alpha(\cdot, f, g)=\alpha\left(\cdot, f^{\prime}, g^{\prime}\right)$ on $[t, s+\delta]$.

We define strategies for player II in a symmetric way and denote by $\mathcal{A}(t)$ (resp. $\mathcal{B}(t))$ the set of strategies for player I (resp. player II).

We define random strategies as before (but with the modified notion of strategies) and still denote by $\mathcal{A}_{r}(t)$ (resp. $\mathcal{B}_{r}(t)$ ) the set of random strategies for player I (resp. player II).

We have an analogue of Lemma 2.1]:

Lemma 5.1 For all $(t, x)$ in $[0, T] \times \mathbb{R}^{n}$, for all $(\alpha, \beta) \in \mathcal{A}(t) \times \mathcal{B}(t)$, there exists a unique couple of controls $(u, v) \in \mathcal{U}(t) \times \mathcal{V}(t)$ that satisfies $P$-a.s.

$$
(u, v)=\left(\alpha\left(\cdot, X^{t, x, u, v}, v .\right), \beta\left(\cdot, X^{t, x, u, v}, u .\right)\right) \text { a.e. on }[t, T] .
$$

One can easily check that the results of the previous parts (i.e., 2.2. Proposition 2.1, Corollary 3.1 and Proposition 3.2) still hold true with the modified notion of strategy. In particular, the game with terminal payoff studied before has a value.

Let us fix $I, J \in \mathbb{N}$. For $1 \leq i \leq I$ and $1 \leq j \leq J$ we consider the terminal cost $g_{i j}: \mathbb{R}^{n} \rightarrow \mathbb{R}$ and the running cost $\ell_{i j}:[0, T] \times \mathbb{R}^{n} \times U \times V \rightarrow \mathbb{R}$ on which we do the following assumptions:

For any $1 \leq i \leq I$ and $1 \leq j \leq J, \ell_{i j}$ and $g_{i j}$ are continuous in all variables, uniformly Lipschitz continuous with respect to $x$ and bounded.

For fixed $(i, j) \in\{1, \ldots, I\} \times\{1, \ldots, J\}$ and strategies $(\alpha, \beta) \in \mathcal{A}(t) \times \mathcal{B}(t)$, we set

$$
J_{i j}(t, x, \alpha, \beta)=E\left[\int_{t}^{T} \ell_{i j}\left(s, X_{s}^{t, x, \alpha, \beta}, \alpha_{s}, \beta_{s}\right) d s+g_{i j}\left(X_{T}^{t, x, \alpha, \beta}\right)\right],
$$

where as before $(\alpha, \beta)$ denotes the unique pair of controls such that (5.24) holds.

The payoff of two random strategies $(\bar{\alpha}, \bar{\beta}) \in \mathcal{A}_{r}(t) \times \mathcal{B}_{r}(t)$, with $\bar{\alpha}=\left(\alpha^{1}, \ldots, \alpha^{R} ; r^{1}, \ldots, r^{R}\right)$ and $\bar{\beta}=\left(\beta^{1}, \ldots, \beta^{S} ; s^{1}, \ldots, s^{S}\right)$, is the average of the payoffs with respect to the probability distributions associated to the strategies:

$$
J_{i j}(t, x, \bar{\alpha}, \bar{\beta})=\sum_{k=1}^{R} \sum_{l=1}^{S} r^{k} s^{l} E\left[\int_{t}^{T} \ell_{i j}\left(s, X_{s}^{t, x, \alpha^{k}, \beta^{l}}, \alpha_{s}^{k}, \beta_{s}^{l}\right) d s+g_{i j}\left(X_{T}^{t, x, \alpha^{k}, \beta^{l}}\right)\right] .
$$


Finally, the payoff of the game is, for $(\hat{\alpha}, \hat{\beta})=\left(\left(\bar{\alpha}_{i}\right)_{1 \leq i \leq I},\left(\bar{\beta}_{j}\right)_{1 \leq j \leq J}\right) \in\left(\mathcal{A}_{r}(t)\right)^{I} \times\left(\mathcal{B}_{r}(t)\right)^{J}$,

$$
J^{p, q}(t, x, \hat{\alpha}, \hat{\beta})=\sum_{i=1}^{I} \sum_{j=1}^{J} p_{i} q_{j} J_{i j}\left(t, x, \bar{\alpha}_{i}, \bar{\beta}_{j}\right) .
$$

We define the value functions for the game with running cost as before by

$$
\begin{aligned}
V^{+}(t, x, p, q) & =\inf _{\hat{\alpha} \in\left(\mathcal{A}_{r}(t)\right)^{I}} \sup _{\left.\hat{\beta} \in \mathcal{B}_{r}(t)\right)^{J}} J^{p, q}(t, x, \hat{\alpha}, \hat{\beta}), \\
V^{-}(t, x, p, q) & =\sup _{\hat{\beta} \in\left(\mathcal{B}_{r}(t)\right)^{J}} \inf _{\hat{\alpha} \in\left(\mathcal{A}_{r}(t)\right)^{I}} J^{p, q}(t, x, \hat{\alpha}, \hat{\beta}) .
\end{aligned}
$$

In our game with running cost, Isaacs' assumption takes the following form: for all $(t, x) \in[0, T] \times \mathbb{R}^{n},(p, q) \in \Delta(I) \times \Delta(J), \xi \in \mathbb{R}^{n}$, and all $A \in \mathcal{S}_{n}:$

$$
\begin{aligned}
\inf _{u} \sup _{v}\left\{<b(t, x, u, v), \xi>+\frac{1}{2} \operatorname{Tr}\left(A \sigma(t, x, u, v) \sigma^{*}(t, x, u, v)\right)-\sum_{i, j} \ell_{i j}(t, x, u, v) p_{i} q_{j}\right\}= \\
\sup _{v} \inf _{u}\left\{<b(t, x, u, v), \xi>+\frac{1}{2} \operatorname{Tr}\left(A \sigma(t, x, u, v) \sigma^{*}(t, x, u, v)\right)-\sum_{i, j} \ell_{i j}(t, x, u, v) p_{i} q_{j}\right\}
\end{aligned}
$$

We set

$$
\begin{aligned}
H(t, x, \xi, A, p, q)= & \inf _{u} \sup _{v}\{\langle b(t, x, u, v), \xi\rangle \\
& \left.+\frac{1}{2} \operatorname{Tr}\left(A \sigma(t, x, u, v) \sigma^{*}(t, x, u, v)\right)-\sum_{i, j} \ell_{i j}(t, x, u, v) p_{i} q_{j}\right\} .
\end{aligned}
$$

Theorem 5.2 Assume that (H), (5.25) and (5.26) hold. Then the game has a value: $V^{+}=V^{-}$, which is the unique solution in the dual sense of

$$
w_{t}+H\left(t, x, D w, D^{2} w, p, q\right)=0
$$

with terminal condition

$$
V^{+}(T, x, p, q)=V^{-}(T, x, p, q)=\sum_{i=1}^{I} \sum_{j=1}^{J} p_{i} q_{j} g_{i j}(x) \quad \forall(x, p, q) \in \mathbb{R}^{n} \times \Delta(I) \times \Delta(J) .
$$

In order to prove Theorem 5.2 it will be convenient to have the following equivalent definition of dual solutions of the Hamilton-Jacobi equation (5.27):

Lemma 5.3 Let $w:[0, T] \times \mathbb{R}^{N} \times \Delta(I) \times \Delta(J) \mapsto \mathbb{R}$ be lower-semicontinuous, uniformly Lipschitz continuous with respect to $p$ and concave with respect to $q$. Then the following statements are equivalent:

(i) $w$ is a dual supersolution of (5.27).

(ii) for any $(\hat{p}, \bar{q}) \in \mathbb{R}^{I} \times \Delta(J)$, for any $\mathcal{C}^{2}$ test function $\phi=\phi(t, x)$ such that

$$
(t, x, p) \mapsto w(t, x, p, \bar{q})-\phi(t, x)-\langle\hat{p}, p\rangle
$$

has a global minimum at some point $(\bar{t}, \bar{x}, \bar{p}) \in[0, T) \times \mathbb{R}^{N} \times \Delta(I)$, we have

$$
\phi_{t}(\bar{t}, \bar{x})+H\left(\bar{t}, \bar{x}, D \phi(\bar{t}, \bar{x}, \bar{p}), D^{2} \phi(\bar{t}, \bar{x}, \bar{p}), \bar{p}, \bar{q}\right) \leq 0 .
$$


Remark : A symmetric statement holds for dual subsolutions.

Proof of Lemma 5.3 : Let us assume that $w$ is a supersolution and let $\phi \in \mathcal{C}^{2}$, $(\hat{p}, \bar{q}) \in \mathbb{R}^{I} \times \Delta(J)$ such that

$$
(t, x, p) \mapsto w(t, x, p, \bar{q})-\phi(t, x)-\langle\hat{p}, p\rangle
$$

has a global minimum at some point $(\bar{t}, \bar{x}, \bar{p}) \in[0, T) \times \mathbb{R}^{N} \times \Delta(I)$. We note that this implies that $\bar{p} \in \partial_{\hat{p}}^{-} w^{*}(\bar{t}, \bar{x}, \hat{p}, \bar{q})$. Moreover, taking the supremum over $p$ in (5.29), we have that $(t, x) \rightarrow-\phi(t, x)-w^{*}(t, x, \hat{p}, \bar{q})$ has a global minimum at $(\bar{t}, \bar{x})$. Since $w^{*}$ is a subsolution of the dual equation, we get

$$
-\phi_{t}+H^{*}\left(\bar{t}, \bar{x},-D \phi,-D^{2} \phi, \bar{p}, \bar{q}\right) \geq 0
$$

at $(\bar{t}, \bar{x})$, because $\bar{p} \in \partial_{\hat{p}}^{-} w^{*}(\bar{t}, \bar{x}, \hat{p}, \bar{q})$. Whence inequality (5.28).

Conversely let us assume that $w$ satisfies (ii). Let $\phi$ be a $\mathcal{C}^{2}$ test function such that $(t, x) \rightarrow w^{*}(t, x, \hat{p}, \bar{q})-\phi(t, x)$ has a maximum at some point $(\bar{t}, \bar{x}) \in(0, T) \times \mathbb{R}^{N}$ for some $(\hat{p}, \bar{q}) \in \mathbb{R}^{I} \times \Delta(J)$. Without loss of generality we can assume that this maximum is a global one. Let $\bar{p} \in \partial_{\hat{p}}^{-} w^{*}(\bar{t}, \bar{x}, \hat{p}, \bar{q})$. ¿From the definition of $w^{*}$, we also have that $(t, x, p) \rightarrow\langle\hat{p}, p\rangle-w(t, x, p, \bar{q})-\phi(t, x)$ has a global maximum at $(\bar{t}, \bar{x}, \bar{p})$, i.e., $(t, x, p) \rightarrow$ $w(t, x, p, \bar{q})+\phi(t, x)-\langle\hat{p}, p\rangle$ has a global minimum at $(\bar{t}, \bar{x}, \bar{p})$. From (5.28) we get

$$
-\phi_{t}+H\left(\bar{t}, \bar{x},-D \phi,-D^{2} \phi, \bar{p}, \bar{q}\right) \leq 0
$$

the desired inequality.

Proof of Theorem 5.2 : $\quad$ Following standard arguments, one first checks that $V^{+}$ and $V^{-}$are globally Hölder continuous, and uniformly Lipschitz continuous with respect to $p$ and $q$. In order to prove other properties of the value functions, let us introduce an extended differential game in $\mathbb{R}^{n+I J}$. This game with asymmetric information and terminal payoff is defined by the dynamics

$$
\begin{aligned}
& d X_{s}=b\left(s, X_{s}, u_{s}, v_{s}\right) d s+\sigma\left(s, X_{s}, u_{s}, v_{s}\right) d B_{s}, s \in[t, T] \\
& d Z_{i j, s}=\ell_{i j}\left(s, X_{s}, u_{s}, v_{s}\right) d s \\
& X_{t}=x, Z_{i j, t}=z_{i j}
\end{aligned}
$$

where $(t, x, z) \in[0, T] \times \mathbb{R}^{n} \times \mathbb{R}^{I J}$, with $z=\left(z_{i j}\right)$, and the terminal $\tilde{g}_{i j}(x, z)=z^{i j}+g_{i j}(x)$. We denote by $\tilde{V}^{+}$and $\tilde{V}^{-}$the upper and lower value of this game. We note that

$$
\tilde{V}^{ \pm}(t, x, z, p, q)=V^{ \pm}(t, x, p, q)+\sum_{i j} z_{i j} p_{i} q_{j}
$$


Following the proofs of Proposition 2.1, one can check that $\tilde{V}^{+}$and $\tilde{V}^{-}$are convex in $p$ and concave in $q$. Hence so are $V^{+}$and $V^{-}$. As in Corollary 3.1 and Proposition 3.2, one can also show that $\tilde{V}^{-}$is a dual supersolution of the HJ equation

$$
\tilde{w}_{t}+\tilde{H}^{-}\left(t, x, z, D_{x, z} w, D_{x}^{2} w\right)=0
$$

where, for $(t, x, z) \in \mathbb{R}^{n+I J}, \xi_{x} \in \mathbb{R}^{n}, \xi_{z} \in \mathbb{R}^{I J}$ and $A \in \mathcal{S}_{n}$,

$$
\begin{aligned}
\tilde{H}^{-}\left(t, x, z, \xi_{x}, \xi_{z}, A\right)= & \sup _{v \in V} \inf _{u \in U}\left\{<b(t, x, u, v), \xi_{x}>\right. \\
& \left.+\frac{1}{2} \operatorname{Tr}\left(A \sigma(t, x, u, v) \sigma^{*}(t, x, u, v)\right)+\sum_{i, j} \ell_{i j}(t, x, u, v) \xi_{z, i j}\right\},
\end{aligned}
$$

while $\tilde{V}^{+}$is a dual subsolution of the HJ equation

$$
\tilde{w}_{t}+\tilde{H}^{+}\left(t, x, z, D_{x, z} w, D_{x}^{2} w\right)=0
$$

where

$$
\begin{aligned}
\tilde{H}^{+}\left(t, x, z, \xi_{x}, \xi_{z}, A\right)= & \inf _{u \in U} \sup _{v \in V}\left\{<b(t, x, u, v), \xi_{x}>\right. \\
& \left.+\frac{1}{2} \operatorname{Tr}\left(A \sigma(t, x, u, v) \sigma^{*}(t, x, u, v)\right)+\sum_{i, j} \ell_{i j}(t, x, u, v) \xi_{z, i j}\right\} .
\end{aligned}
$$

Note that this is precisely at this point that the players have to use the new definition of strategies. Indeed, in order to build their strategies in the sub- and superdynamic programming, they have to compute the running costs $Z_{i j}$ (see the proof of Proposition 3.1). This is possible since, at time $s$, they know the controls $u$. and $v$. and the trajectory $X$. up to time $s-\delta$, and therefore can compute $Z_{i j, s}=z_{i j}+\int_{t}^{s} \ell_{i j}\left(\tau, X_{\tau}, u_{\tau}, v_{\tau}\right) d \tau$.

Using Lemma 5.3 one can then show that $V^{-}$is a dual supersolution of the HJ equation

$$
w_{t}+H^{-}\left(t, x, D w, D^{2} w, p, q\right)=0
$$

where

$$
\begin{aligned}
H^{-}(t, x, \xi, A, p, q)= & \sup _{v \in V} \inf _{u \in U}\left\{<b(t, x, u, v), \xi_{x}>\right. \\
& \left.+\frac{1}{2} \operatorname{Tr}\left(A \sigma(t, x, u, v) \sigma^{*}(t, x, u, v)\right)+\sum_{i, j} \ell_{i j}(t, x, u, v) p_{i} q_{j}\right\}
\end{aligned}
$$

while $V^{+}$is a dual subsolution of the HJ equation

$$
w_{t}+H^{+}\left(t, x, D w, D^{2} w, p, q\right)=0
$$

where

$$
\begin{aligned}
H^{+}(t, x, \xi, A, p, q)= & \inf _{u \in U} \sup _{v \in V}\left\{<b(t, x, u, v), \xi_{x}>\right. \\
& \left.+\frac{1}{2} \operatorname{Tr}\left(A \sigma(t, x, u, v) \sigma^{*}(t, x, u, v)\right)+\sum_{i, j} \ell_{i j}(t, x, u, v) p_{i} q_{j}\right\}
\end{aligned}
$$

Finally combining Isaacs' assumption, which states that $H:=H^{+}=H^{-}$, the fact that $H$ satisfies assumption (4.16) and the comparison principle shows that $V^{+}=V^{-}$is the unique dual solution of (5.27). 


\section{Appendix : A maximum principle}

The following result - used in a crucial way in the proof of the comparison principle - is an adaptation to our framework of the maximum principle for semicontinuous functions (see Theorem 3.2 of [9]):

Theorem 6.1 (Maximum principle) For $k=1,2$, let $\mathcal{O}_{k}$ be open subsets of $\mathbb{R}^{n_{k}}$ and $w_{k}: \mathcal{O}_{k} \times \Delta(I) \times \Delta(J) \rightarrow \mathbb{R}$ be such that

(i) $w_{1}=w_{1}(x, p, q)$ is upper semicontinuous in all variables, convex with respect to $p$ and uniformly Lipschitz continuous with respect to $q$,

(ii) $w_{2}=w_{2}(y, p, q)$ is lower semicontinuous in all its variables, concave with respect to $q$ and uniformly Lipschitz continuous with respect to $p$,

(iii) there is some $\mathcal{C}^{2}$ map $\phi: \mathcal{O}_{1} \times \mathcal{O}_{2} \rightarrow \mathbb{R}$ and some point $(\bar{x}, \bar{y}) \in \mathcal{O}_{1} \times \mathcal{O}_{2}$ such that the map

$$
(x, y) \rightarrow \max _{p, q}\left\{w_{1}(x, p, q)-w_{2}(y, p, q)-\phi(x, y)\right\}
$$

has a maximum at $(\bar{x}, \bar{y})$.

Then, for any $\epsilon>0$, there are $(\bar{p}, \bar{q}) \in \Delta(I) \times \Delta(J),(\hat{p}, \hat{q}) \in \mathbb{R}^{I} \times \mathbb{R}^{J}$ and $\left(X_{1}, X_{2}\right) \in$ $\mathcal{S}_{n_{1}} \times \mathcal{S}_{n_{2}}$ such that the map

$$
(x, y, p, q) \rightarrow w_{1}(x, p, q)-w_{2}(y, p, q)-\phi(x, y)
$$

has a maximum at $(\bar{x}, \bar{y}, \bar{p}, \bar{q})$,

$$
\begin{gathered}
\bar{p} \in \partial_{\hat{p}}^{-} w_{2}^{*}(\bar{y}, \hat{p}, \bar{q}), \bar{q} \in \partial_{\hat{q}}^{-} w_{1}^{\sharp}(\bar{x}, \bar{p}, \hat{q}), \\
\left(-D_{x} \phi(\bar{x}, \bar{y}), X_{1}\right) \in \overline{D^{2,-}} w_{1}^{\sharp}(\bar{x}, \bar{p}, \hat{q}), \quad\left(D_{y} \phi(\bar{x}, \bar{y}), X_{2}\right) \in \overline{D^{2,+}} w_{2}^{*}(\bar{y}, \hat{p}, \bar{q})
\end{gathered}
$$

and

$$
\left(\frac{1}{\epsilon}+\|A\|\right) I \leq\left(\begin{array}{ll}
-X_{1} & 0 \\
0 & X_{2}
\end{array}\right) \leq A+\epsilon A^{2}
$$

with $A=D^{2} \phi(\bar{x}, \bar{y})$.

Remark : Compared with the classical maximum principle, the additional difficulty here is the fact that we need elements of $\overline{D^{2,-}} w_{1}^{\sharp}$ and of $\overline{D^{2,+}} w_{2}^{*}$ while we have only information on the behavior of the difference $w_{1}-w_{2}-\phi$. 
Proof of Theorem 6.1] : We follow closely the proof of Theorem 3.2 of [9]. Let us start by some reductions:

Reductions : As in [9], we can assume without loss of generality that $\mathcal{O}_{k}=\mathbb{R}^{n_{k}}$, $\bar{x}=\bar{y}=0$ and $\phi(x, y)=A(x, y) \cdot(x, y)$ and

$$
\max _{x, y, p, q}\left\{w_{1}(x, p, q)-w_{2}(y, p, q)-\phi(x, y)\right\}=0 .
$$

We can also assume that, for any $\left(\bar{p}^{\prime}, \bar{q}^{\prime}\right) \in \Delta(I) \times \Delta(J)$,

$$
\begin{aligned}
& \text { if }\left(\bar{x}, \bar{y}, \bar{p}^{\prime}, \bar{q}^{\prime}\right) \text { is a maximum point of } w_{1}-w_{2}-\phi \text {, } \\
& \text { then }\left(\bar{p}^{\prime}, \bar{q}^{\prime}\right) \text { belongs to the interior of } \Delta(I) \times \Delta(J) \text {. }
\end{aligned}
$$

Indeed, let us assume that Theorem 6.1 holds true under this additionnal assumption and let us prove that it holds true without. Let

$$
z(x, y, p, q)=w_{1}(x, p, q)-w_{2}(y, p, q)-\phi(x, y)
$$

Among the $(p, q)$ for which $z(\bar{x}, \bar{y}, p, q)$ has a maximum, let us choose $\left(\bar{p}_{0}, \bar{q}_{0}\right)$ such that the total number of indices $i$ and $j$ for which $\left(\bar{p}_{0}\right)_{i}=0$ or $\left(\bar{q}_{0}\right)_{j}=0$ is maximal. Let us denote by $I^{\prime}$ and $J^{\prime}$ the set of indices $i$ and $j$ for which $\left(\bar{p}_{0}\right)_{i}>0$ and $\left(\bar{q}_{0}\right)_{j}>0$. We then define $w_{1}^{\prime}, w_{2}^{\prime}, z^{\prime}, \bar{p}_{0}^{\prime}$ and $\bar{q}_{0}^{\prime}$ as the natural restriction of $w_{1}, w_{2}, z, \bar{p}_{0}$ and $\bar{q}_{0}$ to $\Delta\left(I^{\prime}\right)$ and $\Delta\left(J^{\prime}\right)$. We note that $\left(\bar{x}, \bar{y}, \bar{p}_{0}^{\prime}, \bar{q}_{0}^{\prime}\right)$ is a maximum point of $z$ on $\mathbb{R}^{n_{1}+n_{2}} \times \Delta\left(I^{\prime}\right) \times \Delta\left(J^{\prime}\right)$ and that assumption (6.36) holds, since otherwise one would have a contradiction with the particular choice of $\left(\bar{p}_{0}, \bar{q}_{0}\right)$.

Using now Theorem 6.1 with assumption (6.36), we can build $\left(\bar{p}^{\prime}, \bar{q}^{\prime}\right) \in \Delta\left(I^{\prime}\right) \times \Delta\left(J^{\prime}\right)$, $\hat{q}^{\prime} \in \partial_{q}^{+} w_{1}\left(\bar{x}, \bar{p}^{\prime}, \bar{q}^{\prime}\right), \hat{p}^{\prime} \in \partial_{p}^{-} w_{2}\left(\bar{y}, \bar{p}^{\prime}, \bar{q}^{\prime}\right)$ and $\left(X_{1}, X_{2}\right) \in \mathcal{S}_{n_{1}} \times \mathcal{S}_{n_{2}}$ such that (6.32), (6.33) and (6.34) hold. Then we extend $\left(\bar{p}^{\prime}, \bar{q}^{\prime}\right)$ to $(\bar{p}, \bar{q}) \in \Delta(I) \times \Delta(J)$ by setting $\bar{p}_{i}=\bar{p}_{i}^{\prime}$ for $i \in I^{\prime}$ and $\bar{p}_{i}=0$ otherwise, and $\bar{q}_{j}=\bar{q}_{j}$ for $j \in J^{\prime}$ and $\bar{q}_{j}=0$ otherwise. We also extend $\hat{q}^{\prime}$ to $\hat{q} \in \partial_{q}^{+} w_{1}(\bar{x}, \bar{p}, \bar{q})$ and $\hat{q}^{\prime}$ to $\hat{q} \in \partial_{q}^{+} w_{1}(\bar{x}, \bar{p}, \bar{q})$ by setting $\hat{q}_{j}=M$ for $j \in J \backslash J^{\prime}$ and $\hat{p}_{i}=-M$ for $i \in I \backslash I^{\prime}$, where $M$ is a Lipschitz constant of $w_{1}$ and $w_{2}$ with respect to $q$ and $p$ respectively. This defines $\bar{p}, \bar{q}, \hat{q}, \hat{p}$ and $\left(X_{1}, X_{2}\right)$ for which (6.32), (6.33) and (6.34) hold.

So it remains to prove that Theorem 6.1 holds true under the additional assumption (6.36).

Step 1 : introduction of the inf- and supconvolutions. As in [9], we have

$$
\left(w_{1}\left(x^{\prime}, p, q\right)-\frac{\lambda}{2}\left|x^{\prime}-x\right|^{2}\right)-\left(w_{2}\left(y^{\prime}, p, q\right)-\frac{\lambda}{2}\left|y^{\prime}-y\right|^{2}\right) \leq\left\langle\left(A+\epsilon A^{2}\right)(x, y),(x, y)\right\rangle
$$


for any $\left(x, x^{\prime}, y, y^{\prime}, p, q\right)$, where $\lambda=\frac{1}{\epsilon}+\|A\|$. Let us set for $\lambda^{\prime} \in(0, \lambda)$,

$$
\hat{w}_{1}(x, p, q)=\max _{x^{\prime} \in \mathbb{R}^{n_{1}}, q^{\prime} \in \Delta(J)}\left(w_{1}\left(x^{\prime}, p, q^{\prime}\right)-\frac{\lambda}{2}\left|x^{\prime}-x\right|^{2}-\frac{\lambda^{\prime}}{2}\left|q^{\prime}-q\right|^{2}\right)
$$

and

$$
\hat{w}_{2}(y, p, q)=\min _{y^{\prime} \in \mathbb{R}^{n_{2}}, p^{\prime} \in \Delta(I)}\left(w_{2}\left(y^{\prime}, p^{\prime}, q\right)+\frac{\lambda}{2}\left|y^{\prime}-y\right|^{2}+\frac{\lambda^{\prime}}{2}\left|p^{\prime}-p\right|^{2}\right) .
$$

With these definition we have that $\hat{w}_{1}$ is semiconvex in all its variables with a modulus $\lambda^{\prime}$, semiconvex in $x$ with a modulus $\lambda$ and convex in $p$ (because $w_{1}$ is convex in $p$ by assumption). In the same way, $\hat{w}_{2}$ is semiconcave in all its variables with a modulus $\lambda^{\prime}$, semiconvex in $y$ with a modulus $\lambda$ and concave in $q$ (because $w_{2}$ is concave in $q$ by assumption). Moreover

$$
\hat{w}_{1}(x, p, q)-\hat{w}_{2}(y, p, q)-\left\langle\left(A+\epsilon A^{2}\right)(x, y),(x, y)\right\rangle \leq 0 \quad \forall(x, y, p, q) .
$$

Since $w_{1} \leq \hat{w}_{1}$ and $w_{2} \geq \hat{w}_{2}$, there are some $(p, q)$ such that equality holds in (6.37) at $(0,0, p, q)$. Furthermore, if equality holds at $(0,0, p, q)$, then $(0,0, p, q)$ is a maximum point in (6.35) and assumption (6.36) states that $(p, q)$ belongs to the interior of $\Delta(I) \times \Delta(J)$.

Step 2 : use of Jensen maximum principle. Let us now introduce some small pertubation of the equation: for $\alpha>0$ and $\zeta=\left(\zeta_{x}, \zeta_{y}, \zeta_{p}, \zeta_{q}\right) \in \mathbb{R}^{n_{1}+n_{2}+I+J}$, we set

$$
\begin{array}{r}
z_{\zeta}(x, y, p, q)=\quad \hat{w}_{1}(x, p, q)-\hat{w}_{2}(y, p, q)-\left(A+\epsilon A^{2}\right)(x, y) \cdot(x, y) \\
-\alpha\left(|x|^{2}+|y|^{2}+|p|^{2}-|q|^{2}\right)-\langle\zeta,(x, y, p, q)\rangle .
\end{array}
$$

Note that, because of the penalisation term $\alpha\left(|x|^{2}+|y|^{2}\right)$, for any $\eta>0$, we can choose $\gamma$ small enough such that, for any $\zeta$ such that $|\zeta| \leq \gamma$, any maximum of $z_{\zeta}$ is of the form $(x, y, p, q)$ for some $(x, y) \in B_{\eta}$.

Let $\gamma$ as above. Since $z_{0}$ is semiconvex, has a maximum at $(0,0, p, q)$, Jensen maximum principle (see Lemma A.3 of [9] for instance) states that the set

$$
E_{\gamma}=\left\{\begin{array}{c}
(x, y, p, q) \in B_{\eta} \times \Delta(I) \times \Delta(J), \exists \zeta,|\zeta| \leq \gamma, \text { such that } \\
(i) \quad z_{\zeta} \text { has a maximum at }(x, y, p, q) \text { and } \\
(i i) \quad \hat{w}_{1} \text { and } \hat{w}_{2} \text { have a derivative at }(x, y, p, q)
\end{array}\right\}
$$

has a positive measure. We note that in the quoted Lemma A.3, the maximum is required to be strict ; this assumption is only used in [9] to localize the maximum points, which is not needed here. 
We also note for later use that, if $(x, y, p, q) \in E_{\gamma}$, there is some $\zeta=\left(\zeta_{x}, \zeta_{y}, \zeta_{p}, \zeta_{q}\right)$ with $|\zeta| \leq \gamma$ such that $z_{\zeta}$ has a maximum at $(x, y, p, q)$. In particular, this implies that

$$
q^{\prime} \rightarrow \hat{w}_{1}\left(x, p, q^{\prime}\right)-\hat{w}_{2}\left(y, p, q^{\prime}\right)+\alpha\left|q^{\prime}\right|^{2}-\left\langle\zeta_{q}, q^{\prime}\right\rangle
$$

has a maximum at $q$. Since $\hat{w}_{2}$ is concave in $q, \hat{w}_{1}$ coincides with its concave hull with respect to $q$ at $(x, p, q)$. Hence, if we set $\hat{q}=\frac{\partial \hat{w}_{1}(x, p, q)}{\partial q}$, then

$$
\hat{w}_{1}(x, p, q)+\hat{w}_{1}^{\sharp}(x, p, \hat{q})=q \cdot \hat{q} \text { and } q \in \partial_{\hat{q}}^{+} \hat{w}_{1}^{\sharp}(x, p, \hat{q}) \text {. }
$$

In the same way, if we set $\hat{p}=\frac{\partial \hat{w}_{2}(y, p, q)}{\partial p}$, then we have

$$
\hat{w}_{2}(x, p, q)+\hat{w}_{2}^{*}(y, \hat{p}, q)=p \cdot \hat{p} \text { and } p \in \partial_{\hat{p}}^{-} \hat{w}_{2}^{*}(x, \hat{p}, q) \text {. }
$$

Step 3 : measure estimate of a subset of $E_{\gamma}$. Let $E_{\gamma}^{\prime}$ be the set of points $(x, y, p, q) \in E_{\gamma}$ such that $\hat{w}_{1}^{\sharp}$ has a second order Taylor expansion at $\left(x, p, \frac{\partial \hat{w}_{1}}{\partial q}(x, p, q)\right)$ and $\hat{w}_{2}^{*}$ has a second order Taylor expansion at $\left(y, \frac{\partial \hat{w}_{2}}{\partial p}(x, p, q), q\right)$. Our aim is to show that $E_{\gamma}^{\prime}$ has a full measure in $E_{\gamma}$.

For this we note that $E_{\gamma}^{\prime}=E_{\gamma}^{1} \cap E_{\gamma}^{2}$ where

$$
E_{\gamma}^{1}=\left\{\begin{array}{c}
(x, y, p, q) \in E_{\gamma}, \hat{w}_{1}^{\sharp} \text { has a second order Taylor expansion } \\
\text { at }\left(x, p, \frac{\partial \hat{w}_{1}}{\partial q}(x, p, q)\right)
\end{array}\right\}
$$

and

$$
E_{\gamma}^{2}=\left\{\begin{array}{c}
(x, y, p, q) \in E_{\gamma}, \hat{w}_{2}^{*} \text { has a second order Taylor expansion } \\
\text { at }\left(y, \frac{\partial \hat{w}_{2}}{\partial p}(x, p, q), q\right)
\end{array}\right\}
$$

It is therefore enough to show that $E_{\gamma}^{1}$ and $E_{\gamma}^{2}$ have a full measure in $E_{\gamma}$. We only do the proof for $E_{\gamma}^{1}$, the proof for $E_{\gamma}^{2}$ being symmetric.

Let us set, for any $(x, y, p)$,

$$
E_{\gamma}(x, y, p)=\left\{q \in \Delta(J),(x, y, p, q) \in E_{\gamma}\right\}
$$

and

$$
E_{\gamma}^{1}(x, y, p)=\left\{q \in \Delta(J),(x, y, p, q) \in E_{\gamma}^{1}\right\}
$$

Since $E_{\gamma}$ has a positive measure, from Fubini Theorem we have to show that, for any $(x, y, p)$ such that the set $E_{\gamma}(x, y, p)$ has a positive measure, the set $E_{\gamma}^{1}(x, y, p)$ has a full measure in $E_{\gamma}(x, y, p)$. 
For this, let us introduce the map $\Phi: q \rightarrow \frac{\partial \hat{w}_{1}(x, p, q)}{\partial q}$ defined on $E_{\gamma}(x, y, p)$. We are going to show that

$$
\forall q_{1}, q_{2} \in E_{\gamma}(x, y, p),\left|q_{1}-q_{2}\right| \leq \frac{1}{2 \alpha}\left|\Phi\left(q_{1}\right)-\Phi\left(q_{2}\right)\right|,
$$

which will imply that

$$
\forall E \subset E_{\gamma}(x, y, p) \text { measurable, } \mathcal{L}^{J}(E) \leq \frac{1}{(2 \alpha)^{I}} \mathcal{L}^{J}(\Phi(E)),
$$

where $\mathcal{L}^{J}$ denotes the Lebesgue measure in $\mathbb{R}^{J}$. Then we will prove that (6.41) implies our claim.

Proof of 6.40$)$ : Let $q_{1}, q_{2} \in E_{\gamma}(x, y, p)$. There are $\zeta_{1}$ and $\zeta_{2}$ such that $z_{\zeta_{k}}$ has a maximum at $\left(x, y, p, q_{k}\right)$ for $k=1,2$. The first order optimality conditions imply that

$$
\Phi\left(q_{k}\right)=\frac{\partial \hat{w}_{2}\left(y, p, q_{k}\right)}{\partial q}-2 \alpha q_{k}+\zeta_{k, q} \quad \text { for } k=1,2 .
$$

Using again the optimality of $z_{\zeta_{1}}$ at $q_{1}$ and the fact that $q \rightarrow \hat{w}_{2}(y, p, q)$ is concave, we have

$$
\begin{aligned}
\hat{w}_{1}\left(x, p, q_{2}\right) & \leq \hat{w}_{1}\left(x, p, q_{1}\right)+\left\langle\left(\frac{\partial \hat{w}_{2}\left(y, p, q_{k}\right)}{\partial q}-2 \alpha q_{1}+\zeta_{1, q}\right),\left(q_{2}-q_{1}\right)\right\rangle-\alpha\left|q_{2}-q_{1}\right|^{2} \\
& \leq \hat{w}_{1}\left(x, p, q_{1}\right)+\left\langle\Phi\left(q_{1}\right),\left(q_{2}-q_{1}\right)\right\rangle-\alpha\left|q_{2}-q_{1}\right|^{2}
\end{aligned}
$$

Reversing the role of $q_{1}$ and $q_{2}$ gives

$$
\hat{w}_{1}\left(x, p, q_{1}\right) \leq \hat{w}_{1}\left(x, p, q_{2}\right)+\left\langle\Phi\left(q_{2}\right),\left(q_{1}-q_{2}\right)\right\rangle-\alpha\left|q_{2}-q_{1}\right|^{2}
$$

Adding the two previous inequalities then leads to

$$
0 \leq\left(\Phi\left(q_{2}\right)-\Phi\left(q_{1}\right)\right) \cdot\left(q_{1}-q_{2}\right)-2 \alpha\left|q_{2}-q_{1}\right|^{2}
$$

Whence (6.40).

Proof of 6.41): Let $E$ be a measurable subset of $E_{\gamma}(x, y, p)$. We note that (6.40) states that $\Phi$ is a bijection between $E$ and its image, with a $\frac{1}{2 \alpha}$-Lipschitz continuous inverse. Hence

$$
\mathcal{L}^{I}(E)=\mathcal{L}^{I}\left(\Phi^{-1}(\Phi(E))\right) \leq \frac{1}{(2 \alpha)^{I}} \mathcal{L}^{I}(\Phi(E))
$$

i.e., (6.41) holds.

We finally show that $E_{\gamma}^{1}(x, y, p)$ has a full measure in $E_{\gamma}(x, y, p)$ for any $(x, y, p)$ such that $E_{\gamma}(x, y, p)$ has a positive measure. Let $F$ be the set of $(x, p, \hat{q})$ such that $\hat{w}_{1}^{\sharp}$ has 
a second order Taylor expansion at $(x, p, \hat{q})$. Since $F$ has a full measure, for almost all $(x, p) \in \mathbb{R}^{n} \times \Delta(I)$, the set $F(x, p)=\left\{\hat{q} \in \mathbb{R}^{J},(x, p, \hat{q}) \in F\right\}$ has a full measure in $\mathbb{R}^{J}$. Let $(x, p)$ be such a pair and such that $E_{\gamma}(x, y, p)$ has a positive measure. Then $\Phi\left(E_{\gamma}(x, y, p)\right)$ also has a positive measure from (6.41). Since $\Phi\left(E_{\gamma}(x, y, p)\right) \backslash F(x, p)$ has a zero measure and since

$$
\Phi^{-1}\left(\Phi\left(E_{\gamma}(x, y, p)\right) \backslash F(x, p)\right)=E_{\gamma}(x, y, p) \backslash E_{\gamma}^{1}(x, y, p),
$$

using again (6.41) shows that $E_{\gamma}(x, y, p) \backslash E_{\gamma}^{1}(x, y, p)$ has a zero measure. This completes our claim.

Step 4 : (further) magic properties of sup-convolution. We now explain that one can use second order Taylor expansions of $\hat{w}_{1}^{\sharp}$ and $\hat{w}_{2}^{*}$ to get elements of $D^{2,-} w_{1}^{\sharp}, D^{2,+} \hat{w}_{1}$, $D^{2,+} w_{2}^{*}$ and $D^{2,-} \hat{w}_{1}$.

¿From our assumption (6.36), we know that, for $\epsilon$ small enough, if $(0,0, p, q)$ realizes the equality in (6.37), then $(p, q)$ belongs to the interior of $\Delta(I) \times \Delta(J)$. Hence we can find $\alpha, \gamma>0$ so small that, for any $\zeta$ with $|\zeta| \leq \gamma$, if $(x, y, p, q)$ realizes the maximum of $z_{\zeta}$, then $(p, q)$ belongs to the interior of $\Delta(I) \times \Delta(J)$.

Let us now fix $\gamma>0$ small enough and let us compute $\hat{w}_{1}^{\sharp}$ at $(x, p, \hat{q})$ for $(x, y, p, q) \in E_{\gamma}^{\prime}$ and $\hat{q}=\frac{\partial \hat{w}_{1}(x, p, q)}{\partial q}$. We have

$$
\left.\hat{w}_{1}^{\sharp}(x, p, \hat{q})=\min _{x^{\prime}, q^{\prime}, q^{\prime \prime}}\left(q^{\prime} \cdot \hat{q}+\frac{\lambda}{2}\left|x^{\prime}-x\right|^{2}+\frac{\lambda^{\prime}}{2}\left|q^{\prime \prime}-q^{\prime}\right|^{2}\right)-w_{1}\left(x^{\prime}, p, q^{\prime \prime}\right)\right)
$$

¿From (6.38), we have that $\hat{w}_{1}(x, p, q)+\hat{w}_{1}^{\sharp}(x, p, \hat{q})=q . \hat{q}$ and $q \in \partial_{\hat{q}}^{+} \hat{w}_{1}^{\sharp}(x, p, \hat{q})$. In particular, $q^{\prime}=q$ is a minimum point in (6.42). Since $q$ belongs to the interior of $\Delta(J)$, the optimality conditions imply that, if $\left(x^{\prime}, q, q^{\prime \prime}\right)$ is a minimum of (6.42), then $q=q^{\prime \prime}-\frac{1}{\lambda^{\prime}} \hat{q}$. Therefore

$$
\begin{aligned}
\hat{w}_{1}^{\sharp}(x, p, \hat{q}) & =-\frac{1}{2 \lambda^{\prime}}|\hat{q}|^{2}+\min _{x^{\prime}, q "}\left(q^{\prime \prime} \cdot \hat{q}-w_{1}\left(x^{\prime}, p, q^{\prime \prime}\right)+\frac{\lambda}{2}\left|x^{\prime}-x\right|^{2}\right) \\
& =-\frac{1}{2 \lambda^{\prime}}|\hat{q}|^{2}+\min _{x^{\prime}}\left(w_{1}^{\sharp}\left(x^{\prime}, p, \hat{q}\right)+\frac{\lambda}{2}\left|x^{\prime}-x\right|^{2}\right),
\end{aligned}
$$

In particular, $q " \in \partial_{\hat{q}}^{+} w_{1}\left(x^{\prime}, p, \hat{q}\right)$, which shows that

$$
q+\frac{1}{\lambda^{\prime}} \hat{q} \in \partial_{\hat{q}}^{+} w_{1}^{\sharp}(x+\xi / \lambda, p, \hat{q})
$$

Moreover, $x \rightarrow \hat{w}_{1}^{\sharp}(x, p, \hat{q})$ is equal, up to a constant, to the inf-convolution of $w_{1}^{\sharp}$ with respect to $x$. Since $\hat{w}_{1}^{\sharp}$ has a second order Taylor expansion in $x$ at such a point $(x, p, \hat{q})$, the classical "magic properties" of inf-convolution (see Lemma A.4 of [9]) state that $x^{\prime}=x+\xi / \lambda$ and

$$
\left(D \hat{w}_{1}^{\sharp}(x, p, \hat{q}), D^{2} \hat{w}_{1}^{\sharp}(x, p, \hat{q})\right) \in D^{2,-} w_{1}^{\sharp}(x+\xi / \lambda, p, \hat{q}) .
$$


where $\xi=D \hat{w}_{1}^{\sharp}(x, p, \hat{q})$.

Following [1] we also note that for any $x^{\prime}$ close to $x$, we have

$$
\hat{w}_{1}\left(x^{\prime}, p, q\right) \leq q \cdot \hat{q}-\hat{w}_{1}^{\sharp}\left(x^{\prime}, p, \hat{q}\right)=\hat{w}_{1}(x, p, \hat{q})+\hat{w}_{1}^{\sharp}(x, p, \hat{q})-\hat{w}_{1}^{\sharp}\left(x^{\prime}, p, \hat{q}\right)
$$

because $\hat{w}_{1}(x, p, q)+\hat{w}_{1}^{\sharp}(x, p, \hat{q})=q . \hat{q}$. Since $\hat{w}_{1}^{\sharp}$ has a second order Taylor expansion at $x$, this gives

$$
-\left(D \hat{w}_{1}^{\sharp}(x, p, \hat{q}), D^{2} \hat{w}_{1}^{\sharp}(x, p, \hat{q})\right) \in D^{2,+} \hat{w}_{1}(x, p, q) .
$$

In a symmetric way, if $(x, y, p, q) \in E_{\gamma}^{\prime}$ and $\hat{p}=\frac{\partial \hat{w}_{2}(y, p, q)}{\partial p}$, then

$$
\left(D \hat{w}_{2}^{*}(y, \hat{p}, q), D^{2} \hat{w}_{2}^{*}(y, \hat{p}, q)\right) \in D^{2,+} w_{2}^{*}(y+\xi / \lambda, \hat{p}, q),
$$

where $\xi=D \hat{w}_{2}^{*}(y, \hat{p}, q)$,

$$
p+\frac{1}{\lambda^{\prime}} \hat{p} \in \partial_{\hat{p}}^{-} w_{2}^{*}(y+\xi / \lambda, \hat{p}, q)
$$

and

$$
-\left(D \hat{w}_{2}^{*}(y, \hat{p}, q), D^{2} \hat{w}_{2}^{*}(y, \hat{p}, q)\right) \in D^{2,-} \hat{w}_{2}(y, p, q) .
$$

Step 5 : conclusion. From the previous steps, we know that the set $E_{\gamma}^{\prime}$ defined in step 3 has a positive measure for any $\alpha, \gamma>0$ sufficiently small. Hence we can find sequences $\lambda_{n}^{\prime} \rightarrow+\infty, \alpha_{n}, \gamma_{n} \rightarrow 0^{+}, \zeta_{n}=\left(\zeta_{x}^{n}, \zeta_{y}^{n}, \zeta_{p}^{n}, \zeta_{q}^{n}\right) \rightarrow 0,\left(x_{n}, y_{n}, p_{n}, q_{n}\right)$ converging to some $(0,0, \bar{p}, \bar{q})$ such that $\left(x_{n}, y_{n}, p_{n}, q_{n}\right) \in E_{\gamma}^{\prime}$ and such that the map $z_{\zeta_{n}}$ has a maximum at $\left(x_{n}, y_{n}, p_{n}, q_{n}\right)$.

Let us set

$$
\begin{aligned}
& \hat{p}_{n}=\frac{\partial \hat{w}_{2}\left(y, p_{n}, q_{n}\right)}{\partial p}, \quad \hat{q}_{n}=\frac{\partial \hat{w}_{1}\left(x, p_{n}, q_{n}\right)}{\partial q}, \\
& \left(\xi_{1}^{n}, X_{1}^{n}\right)=\left(D \hat{w}_{1}^{\sharp}\left(x_{n}, p_{n}, \hat{q}_{n}\right), D^{2} \hat{w}_{1}^{\sharp}\left(x_{n}, p_{n}, \hat{q}_{n}\right)\right)
\end{aligned}
$$

and

$$
\left(\xi_{2}^{n}, X_{2}^{n}\right)=\left(D \hat{w}_{2}^{*}\left(y_{n}, \hat{p}_{n}, q_{n}\right), D^{2} \hat{w}_{2}^{*}\left(y_{n}, \hat{p}_{n}, q_{n}\right)\right) .
$$

¿From (6.43) and (6.47) we have

$$
p_{n}+\frac{1}{\lambda_{n}^{\prime}} \hat{p}_{n} \in \partial_{\hat{p}}^{-} w_{2}^{*}\left(y_{n}+\xi_{2}^{n} / \lambda, \hat{p}_{n}, q_{n}\right) \text { and } q_{n}+\frac{1}{\lambda_{n}^{\prime}} \hat{q}_{n} \in \partial_{\hat{q}}^{+} w_{1}^{\sharp}\left(x_{n}+\xi_{n}^{1} / \lambda, p_{n}, \hat{q}_{n}\right) .
$$

¿From (6.45) and (6.48) we have

$$
-\left(\xi_{1}^{n}, X_{1}^{n}\right) \in D^{2,+} \hat{w}_{1}\left(x_{n}, p_{n}, q_{n}\right) \text { and }-\left(\xi_{2}^{n}, X_{2}^{n}\right) \in D^{2,-} \hat{w}_{2}\left(x_{n}, p_{n}, q_{n}\right),
$$

Since furthermore $(x, y) \rightarrow z_{\zeta_{n}}\left(x, y, p_{n}, q_{n}\right)$ has a maximum at $\left(x_{n}, y_{n}, p_{n}, q_{n}\right)$, the first and second order optimality conditions imply that

$$
\left(-\xi_{1}^{n}, \xi_{2}^{n}\right)=\left(A+\epsilon A^{2}\right)\left(x_{n}, y_{n}\right)+2 \alpha_{n}\left(x_{n}, y_{n}\right)+\left(\zeta_{x}^{n}, \zeta_{y}^{n}\right)
$$


and

$$
\left(\frac{1}{\epsilon}+\|A\|\right) I \leq\left(\begin{array}{ll}
-X_{1}^{n} & 0 \\
0 & X_{2}^{n}
\end{array}\right) \leq A+\epsilon A^{2}+2 \alpha_{n} I
$$

The left-hand side inequality is due to the fact that $\hat{w}_{1}$ and $\hat{w}_{2}$ are semiconvex and semiconcave w.r. to $x$ and $y$ respectively with a modulus $\lambda=\frac{1}{\epsilon}+\|A\|$. Using (6.44) and (6.46) gives

$$
\left(\xi_{1}^{n}, X_{1}^{n}\right) \in D^{2,-} w_{2}^{\sharp}\left(x_{n}+\xi_{1}^{n} / \lambda, p_{n}, \hat{q}_{n}\right) \text { and }\left(\xi_{2}^{n}, X_{2}^{n}\right) \in D^{2,+} w_{1}^{*}\left(y_{n}+\xi_{2}^{n} / \lambda, \hat{p}_{n}, q_{n}\right)
$$

We now note that $\left(X_{1}^{n}\right),\left(X_{2}^{n}\right),\left(\hat{p}_{n}\right)$ and $\left(\hat{q}_{n}\right)$ are bounded. For $\left(X_{1}^{n}\right),\left(X_{2}^{n}\right)$ this is an obvious consequence of (6.52). For $\left(\hat{p}_{n}\right)$ and $\left(\hat{q}_{n}\right)$ this comes from (6.49), from the Lipschitz continuity assumption of $w_{2}$ and $w_{1}$ with respect to $p$ and $q$ respectively and from the definition of $\hat{w}_{1}$ and $\hat{w}_{2}$.

We now let $n \rightarrow+\infty$. From (6.51), we have $\xi_{1}^{n}, \xi_{2}^{n} \rightarrow 0$. We can assume that $\left(\hat{p}_{n}, \hat{q}_{n}\right) \rightarrow$ $(\hat{p}, \hat{q}), X_{1}^{n} \rightarrow X_{1}$ and $X_{2}^{n} \rightarrow X_{2}$. Then we have from (6.50), (6.53) and (6.52) that:

$$
\begin{gathered}
\bar{p} \in \partial_{\hat{p}}^{-} w_{2}^{*}(0, \hat{p}, \bar{q}) \text { and } \bar{q} \in \partial_{\hat{q}}^{+} w_{1}^{\sharp}(0, \bar{p}, \hat{q}), \\
\left(0, X_{1}\right) \in \overline{D^{2,-}} w_{2}^{\sharp}(0, \bar{p}, \hat{q}) \text { and }\left(0, X_{2}\right) \in \overline{D^{2,+}} w_{1}^{*}(0, \hat{p}, \bar{q})
\end{gathered}
$$

and

$$
\left(\frac{1}{\epsilon}+\|A\|\right) I \leq\left(\begin{array}{ll}
-X_{1} & 0 \\
0 & X_{2}
\end{array}\right) \leq A+\epsilon A^{2} .
$$

\section{References}

[1] Alvarez, O.; Lasry J.-M.; Lions P.-L. (1997) Convex viscosity solutions and state-constraints. J. Math. Pures Appl. 9, 76(3), 265-288.

[2] Amendinger, D.; Becherer, A.; Schweizer, M. (2003) A monetary value for initial information in portfolio optimization. Finance and Stochastics 7(1) 29-46.

[3] Aumann, R. J.; Maschler, M. B. Repeated games with Incomplete InforMation. With the collaboration of Richard E. Stearns. MIT Press, Cambridge, MA, 1995.

[4] Bensoussan, A. and Frehse, J. (2000) Stochastic games for $N$ players. J. Optimization Theory Appl. 105, No.3, 543-565. 
[5] Cardaliaguet P. (2006) Differential games with asymmetric information. To appear in SIAM J. Control Optim.

[6] Cardaliaguet P. (2006) Representation formulas for differential games with asymmetric information. To appear in JOTA.

[7] Corcuera, J.M.; Imkeller, P.; Kohatsu-Higa, A.; Nualart, D. (2004) Additional utility of insiders with imperfect dynamical information. Finance and Stochastics $8437-450$.

[8] Crandall M.G., Lions P.-L. Viscosity solutions of Hamilton-Jacobi Equations. Trans. Amer. Math. Soc. (1983), 277, 1-42.

[9] Crandall M.G., Ishii H., Lions P.-L. (1992) User's guide to viscosity solutions of second order Partial Differential Equations. Bull. Amer. Soc., 27, pp. 1-67.

[10] De Meyer, B. Repeated games, duality and the central limit theorem. Math. Oper. Res. 21 (1996), no. 1, 237-251.

[11] De Meyer, B.; Rosenberg, D. "Cav u" and the dual game. Math. Oper. Res. 24 (1999), no. 3, 619-626.

[12] Fleming, W.H.; Souganidis, P.E. On the existence of value functions of twoplayer, zero-sum stochastic differential games. Indiana Univ. Math. J. 38 (1989), No.2, 293-314.

[13] Mertens, J.F.; Zamir S. The value of two person zero sum repeated games with lack of information on both sides, Int. J. Game Theory (1994), 1, pp. 39-64.

[14] Nisio, M. (1988) Stochastic differential games and viscosity solutions of Isaacs equations. Nagoya Math. J. 110, 163-184 (1988)

[15] Rosenberg; Solan E.; Vieille N. Stochastic games with a singler controller and incomplete information. SIAM J. Control Optim. Vol. 43 (2004), N. 1, pp. 86110

[16] Sorin, S. A first course on Zero-sum repeated games. Mathématiques \& Applications (Berlin), 37. Springer-Verlag, Berlin, 2002. 